\title{
Targeting ischemia-induced KCC2 hypofunction rescues refractory neonatal seizures and mitigates epileptogenesis in a mouse model
}

Running title: KCC2 hypofunction in neonatal ischemic seizures

Number of words: 10606

Number of pages: 60

Number of Figures: 8

Number of Supplemental Figures: 4

Resources Table: 1

Keywords: hypoxic-ischemic encephalopathy, refractory, neonatal seizures, KCC2 hypofunction, epilepsy

Abbreviations: Phenobarbital (PB), Antiseizure medication (ASM), Electroencephalography (EEG), K-Cl co-transporter 2 (KCC2), Na-K-Cl cotransporter 1 (NKCC1), GABA receptor (GABAR) hypoxic-ischemic encephalopathy (HIE) Tropomyosin receptor kinase $B($ TrkB)

\section{Corresponding Author:}

Shilpa D. Kadam, PhD

Neuroscience Laboratory, Hugo Moser Research Institute at Kennedy Krieger; Department of Neurology, Johns Hopkins University School of Medicine, 707 North Broadway, $400 \mathrm{H}$;

Baltimore, MD 21205

Phone: 443-923-2688, Fax: 443-923-2695, E-mail: kadam@kennedykrieger.org 


\section{Abstract}

Neonatal seizures pose a clinical challenge for their early detection, acute management, and mitigation of long-term comorbidities. A major cause of neonatal

49 seizures is hypoxic-ischemic encephalopathy that results in seizures that are frequently

50 refractory to the first-line anti-seizure medication phenobarbital (PB). One proposed

51 mechanism for PB-inefficacy during neonatal seizures is the reduced expression and

52 function of the neuron-specific $\mathrm{K}^{+} / \mathrm{Cl}^{-}$cotransporter 2 (KCC2), the main neuronal $\mathrm{Cl}^{-}$

53 extruder that maintains chloride homeostasis and influences the efficacy of GABAergic

54 inhibition. To determine if PB-refractoriness after ischemic neonatal seizures is

55 dependent upon KCC2 hypofunction and can be rescued by KCC2 functional

56 enhancement, we investigated the recently developed KCC2 functional enhancer

57 CLP290 in a CD-1 mouse model of refractory ischemic neonatal seizures quantified with

58 vEEG. We report that acute CLP290 intervention can rescue PB-resistance, KCC2

59 expression, and the development of epileptogenesis after ischemic neonatal seizures.

60 KCC2 phosphorylation sites have a strong influence over KCC2 activity and seizure

61 susceptibility in adult experimental epilepsy models. Therefore, we investigated seizure

62 susceptibility in two different knock-in mice in which either phosphorylation of S940 or

63 T906/T1007 was prevented. We report that KCC2 phosphorylation regulates both

64 neonatal seizure susceptibility and CLP290-mediated KCC2 functional enhancement.

65 Our results validate KCC2 as a clinically relevant target for refractory neonatal seizures

66 and provide insights for future KCC2 drug development. 


\section{Introduction}

Neonatal seizures occur in an estimated 1 to 3.5 per 1000 live births in the term

71 infant. Hypoxic-ischemic encephalopathy (HIE) is a major cause of acute neonatal

72 seizures (1). The management of these seizures are a major clinical challenge as they

73 are often refractory to an initial loading dose of the first-line anti-seizure medication (ASM)

74 phenobarbital (PB) and adjunct $\operatorname{ASMs}(2,3)$. Compared to seizures at older ages,

75 neonatal seizures differ in their etiology, semiology, electrographic signature, and

76 response to $\operatorname{ASMs}(1,4)$. There is an urgent need to identify the developmental

77 mechanisms underlying seizure susceptibility and ineffective ASM response in the

78 neonatal brain.

79 The neonatal brain has lower expression of its chief chloride extruder the neuron-

80 specific $\mathrm{K}^{+}-\mathrm{Cl}^{-}$cotransporter 2 (KCC2), and a high neuronal intracellular chloride

81 concentration $\left(\left[\mathrm{Cl}^{-}\right]_{i}\right)(5-7)$. GABA is the primary inhibitory neurotransmitter in the mature

82 brain, however in the neonatal brain the activation of GABAA receptors (GABARs) results

83 in depolarizing actions on immature neurons (8). KCC2 expression and function increase

84 during development, resulting in a lower $\left[\mathrm{Cl}^{-}\right]$i that coincides with a developmental shift

85 from depolarizing to hyperpolarizing GABAergic signaling. The importance of KCC2

86 function in seizure susceptibility is supported by emerging evidence from human genetics,

87 as pathogenic variants in SLC12A5 are associated with the development of idiopathic

88 generalized epilepsy and early infantile epileptic encephalopathy (OMIM \#616685 and

$89 \quad$ \#616645, respectively) (9).

90 Despite the introduction of many new ASMs into clinical practice over the past 20

91 years, the incidence of refractory seizures has remained unchanged (10). KCC2 
92 hypofunction is increasingly associated with pharmaco-resistant epilepsies and is a

93 proposed cause of disinhibition $(11,12)$. GABAAR mediated fast synaptic inhibition and

94 the anti-seizure efficacy of PB (a positive allosteric modulator of GABAARs) are both

95 dependent upon active neuronal $\mathrm{Cl}^{-}$extrusion $(13,14)$. In a preclinical CD-1 mouse model

96 of ischemic neonatal seizures, KCC2 is transiently downregulated and associated with

97 PB-resistant seizures (15). In this model, post-ischemic tropomyosin receptor kinase

98 (TrkB) inhibition rescued PB-resistant neonatal seizures and KCC2 expression (16, 17).

99 This acute rescue of KCC2 hypofunction via TrkB inhibition improved long-term outcomes

100 after ischemic neonatal seizures $(18,19)$. These results suggest KCC2 as a novel

101 therapeutic target for refractory neonatal seizures.

102 A recent high throughput screen for compounds that reduce $\left[\mathrm{Cl}^{-}\right]$in a cell line with low 103 KCC2 expression identified the compound CLP257 as a KCC2 functional enhancer (20).

104 To improve the poor pharmacokinetics of CLP257, the carbamate prodrug CLP290 was 105 designed and improved the half-life ( $\left.\mathrm{t}_{1 / 2}\right)$ from $<15 \mathrm{~min}$ to $5 \mathrm{~h}(20)$. These compounds 106 provide an opportunity to test whether KCC2 functional enhancement can rescue the 107 emergence of PB-resistant neonatal seizures after ischemia in our preclinical CD-1 108 mouse model. KCC2 expression increases twofold in mouse pups during development 109 between P7 and P10 (15). Therefore to examine the developmental differences in 110 ischemic seizure suppression after KCC2 functional enhancement both age groups were

111 included. Neonatal seizures are associated with poor long-term outcomes including the 112 development of epilepsy $(21,22)$. Therefore, the effect of acute CLP290 intervention at 113 P7 was evaluated at P12 using a new subthreshold pentylenetetrazol (PTZ) dosing 114 protocol to investigate epileptogenesis. To test if KCC2 hypofunction could induce ictal 
115 events independent of ischemia, the selective KCC2 inhibitor VU0463271 was

116 administered to P7 naïve pups during vEEG. KCC2 posttranslational modifications are

117 tightly regulated throughout development and strongly influence KCC2 activity (23-25).

118 The KCC2 phosphorylation sites S940 (25) and T1007 (24) were investigated for their

119 role in CLP290-mediated effects on neonatal refractory seizures. Using two knock-in

120 mutant mice that prevent either S940 (25) or T1007 (24) phosphorylation, we investigated

121 the importance of these sites on neonatal seizure susceptibility and post-ischemic PB-

122 efficacy.

\section{Results}

124 CLP290 rescues phenobarbital-resistant seizures at P7

125 The true burden of acute ischemic seizures in mouse pups cannot be identified by

126 behavioral scoring parameters alone and require continuous VEEG recordings as their

127 presentation can range from entirely electrographic to generalized convulsive (7). In our

128 CD-1 neonatal mouse model of unilateral carotid ligation, pups presented with PB-

129 resistant ischemic neonatal seizures at P7 (Fig. 1A-D), an established characteristic of

130 the model $(15-17,26)$. All pups underwent $1 \mathrm{~h}$ of baseline $\mathrm{VEEG}$ recording after unilateral

131 carotid ligation. After $1 \mathrm{~h}$ of $\mathrm{vEEG}$ recording all pups received a loading dose of PB with a

132 subsequent hour of vEEG recording (Fig. 1A-D). Pups that only received a PB loading

133 dose (PB-only) were pharmacoresistant, as their $2{ }^{\text {nd }} \mathrm{h}$ seizure burden remained high and

134 was similar to $1^{\text {sth }}$ levels (Fig 1C-F).

135 To investigate if the KCC2 functional enhancer CLP290 could rescue PB-resistant

136 seizures, CLP290 was administered intraperitoneally at P7 in the Pre, Post, or Primed

137 groups (Fig. 1 B). Administration of Primed CLP290 10mg/kg (10') significantly reduced 
138 total seizure burden, duration, and frequency of $2^{\text {nd } h}$ seizure events (Fig. 1C-E). This

139 decrease in $2^{\text {nd }} \mathrm{h}$ seizure burden significantly increased seizure suppression after PB

140 administration, thereby rescuing P7 PB-resistance (Fig. 1D-F). In contrast to 10', bolus

141 administration of $20 \mathrm{mg} / \mathrm{kg}$ CLP290 reduced $1^{\text {sth }}$ baseline seizure burden (Supplemental

142 Fig. 1).

143

144 Pro-drug CLP290 improved brain availability

145 A major limiting factor for epilepsy drug development is the in vivo brain availability 146 of candidate compounds identified in vitro $(27,28)$. CLP290 is a carbamate prodrug of

147 CLP257 and has an improved $t_{1 / 2}$ from <15min (CLP257) to 5h (CLP290) in blood samples

148 from adult rats (20). The brain availability of CLP257 and CLP290 have not been

149 previously published. At P7, CLP257 was unable to reduce $2^{\text {nd }}$ h seizure burden (Fig. 1

150 G-J) when administered systemically at the 10' dose. To determine if CLP290 efficacy

151 and CLP257 inefficacy were due to differences in pharmacokinetics, we performed HPLC

152 to analyze brain levels of CLP257 after I.P. delivery of either CLP257 or CLP290 (Fig. 1K

153 and Supplemental Fig. 2). At P7 and P10, CLP290 administration of 10 or 20mg/kg

154 demonstrated adequate brain availability. (Fig. 1L). CLP257 had poor brain availability

155 compared to CLP290 (Fig. 1K-M), which suggested that the inability of CLP257 to rescue

156 PB-refractory seizures was due to its poor pharmacokinetic profile.

CLP290 efficacy on phenobarbital-responsive seizures at P10

As previously reported $(15-17,26)$, ischemic neonatal seizures after unilateral

160 carotid ligation in P10 CD-1 pups were PB-responsive (Fig. 2 A-E). The age-dependent 
161 emergence of PB-resistant and PB-responsive seizures at P7 versus P10 is a

162 characteristic of the neonatal mouse model that is associated with a twofold increase in

163 KCC2 expression between P7 and P10 (15). Administration of CLP290 at P10 did not

164 further improve the efficacy of PB at any of the doses tested (Fig. 2 C-E). When ischemic

165 neonatal seizures were PB-responsive at P10, the previously reported TrkB-antagonist

166 ANA12 $(17,26)$ also did not improve the efficacy of PB. This suggests that the therapeutic

167 benefit of KCC2 functional enhancement is dependent upon the degree of KCC2

168 hypofunction which is evident in the emergence of refractoriness at P7 but not at P10.

CLP290 rescued ipsilateral post-ischemic KCC2 and $\$ 940$ downregulation

PB-resistant ischemic neonatal seizures have been shown to significantly reduce

172 expression of KCC2 and phosphorylation of S940 24h after ischemia (17). P7 unilateral

173 carotid ligation did not result in an infarct stroke injury, therefore KCC2 degradation at

$17424 \mathrm{~h}$ is not caused by infarct related cell-death (15). In this model, KCC2 expression

175 undergoes a recovery over 3-4 days, a characteristic that is associated with the transient

176 nature of neonatal seizures due to $\operatorname{HIE}(29,30)$. At 24h after P7 unilateral carotid ligation,

177 KCC2 and S940 expression was significantly lower in the right hemisphere (ipsilateral to

178 ischemia) than the left hemisphere (contralateral to ischemia) in the PB-only group (Fig.

1793 A-E). Intervention with CLP290 at P7 rescued both KCC2 and S940 downregulation in

180 the 10' group 24h after ligation (Fig. 3 D-E). Bolus administration of CLP290 20mg/kg

181 Post significantly increased S940 expression bilaterally compared to the PB-only group

182 at P7 (Fig. 3C and Supplemental Fig. 3 A-B). When ischemic neonatal seizures are PB-

183 responsive at P10, KCC2 expression was not significantly decreased at 24h (Fig. 3 F-I). 
184 However, the ratio of ipsilateral to contralateral S940 expression in the PB-only group

185 was significantly decreased (Fig. 3 H-J) and was rescued in all CLP290 treatment groups

186 (Fig. $3 \mathrm{H}-\mathrm{J})$. Therefore, when ischemic neonatal seizures were PB-resistant, CLP290

187 rescued KCC2 expression and S940 phosphorylation, suggesting that the degree of

188 KCC2 hypofunction drives the therapeutic benefit of KCC2 functional enhancement.

CLP290 rescue of PB-resistant seizures was not mediated through BDNF-TrkB Ischemic neonatal seizures at P7 induce a significant bilateral increase in TrkB 192 expression and phosphorylation of Y816 (17, 26). Similarly, TrkB expression and Y816

193 phosphorylation were significantly upregulated bilaterally in the PB-only group $24 \mathrm{~h}$ after 194 ischemic neonatal seizures at P7 (Fig. 3K-O) but not at P10 (Fig. 3 P-T and Supplemental 195 Fig. 3 C-D). In this study, the bilateral increase in post-ischemic TrkB and Y816 196 expression was detected as previously characterized (17). Increased TrkB expression 197 was not significantly rescued by CLP290 however Y816 was (Fig. 3 K-O). 24h after P7, 198 the Y816/TrkB ratios demonstrated a significant bilateral reduction in the ratio by CLP290 19920 post (Supplemental Fig. 3C), driven by the significant increase in TrkB expression (Fig.

200 3L-O). In this model, ischemic neonatal seizures at P10 were PB-responsive and P10 201 pups did not show activation of TrkB (Fig. 3P-T and Supplemental Fig. 3 C-D). These 202 results suggest that the CLP290-mediated seizure suppression in the 10' group at P7 was 203 independent of TrkB. 
HIE seizures are transient within the first week of life $(29,30)$ and the long-term

207 consequences of neonatal seizures are difficult to isolate from the consequences of

208 prolonged and/or inefficacious anti-seizure therapy in the clinic (22). In our CD-1 mouse

209 model of refractory neonatal ischemic seizures, we have previously documented the

210 emergence of epilepsy in adulthood (31). To assess if the acute rescue of PB-resistant

211 neonatal seizures at P7 via CLP290 had long-term benefits, the same pups underwent a

212 PTZ challenge at P12 (Fig. 4A-B). Previously, a $80 \mathrm{mg} / \mathrm{kg}$ dose of PTZ was shown to

213 induce high seizure burdens that were PB-responsive and upregulated KCC2 at P7 in

214 CD-1 pups (32). In this study, pups were administered three doses of PTZ (20, 20, and

$21540 \mathrm{mg} / \mathrm{kg}$ ) 1h apart (Fig. 4A-B). Using this protocol, we characterized seizure

216 susceptibility in P7 naïve, P7 PB-only, and P7 CLP290 10' treated pups at P12. The initial

217 dose of PTZ induced seizures in the $1^{\text {sth }}$ for all groups (Fig. 4A-D). Naïve pups

218 demonstrated a general reduction in seizures during the second and third PTZ doses,

219 potentially uncovering a homeostatic compensation to help develop resistance to the

220 chemoconvulsant induced seizures. PB-only pups demonstrated an increase in seizure

221 burden after the repeated doses of PTZ, with mice $(n=3)$ going into status epilepticus.

222 Thus, the significant increase in PB-only seizure burdens at P12 was driven by a

223 significant increase in seizure duration (Fig. 4E). CLP290 10' treated pups had similar

224 P12 seizure burdens as naïve pups and significantly lower than PB-only pups (Fig. 4A-

225 D). This novel PTZ challenge protocol identified epileptogenesis in the form of heightened

226 susceptibility to seizures at P12 for neonatal pups that underwent standard but

227 inefficacious PB treatment for their refractory seizures, efficacious CLP290 10'

228 intervention at P7 resulted in the regression of epileptogenesis at P12. 
In vivo KCC2 inhibition is epileptogenic in the neonatal brain

230 The selective KCC2 inhibitor VU0463271 (VU) has been shown to induce

231 epileptiform discharges in the dorsal hippocampus of the adult mouse, highlighting the 232 critical role of KCC2 in the mature hippocampus (33). Here, naive P7 CD-1 pups were

233 administered $0.25 \mathrm{mg} / \mathrm{kg}$ VU (I.P.) at the initiation of vEEG recording with a subsequent

234 dose of $0.5 \mathrm{mg} / \mathrm{kg}$ VU (I.P.) at $1 \mathrm{~h}$ (Fig. 5A-B). Selective inhibition of KCC2 was sufficient

235 to induce epileptiform activity (Fig. 5A-E) at P7. Prolonged and repeated seizures are

236 known to play a role in the reduction of neuronal surface KCC2 expression and function

237 (15, 34-36). At P7, if post-ischemic KCC2 hypofunction plays a critical role in PB-

238 refractoriness, KCC2 inhibition following repeated ischemic seizures would be expected

239 to further aggravate the seizure burden. To test the effect of KCC2 inhibition following

240 repeated ischemia-induced neonatal seizures at P7, VU $0.25 \mathrm{mg} / \mathrm{kg}$ (I.P.) was

241 administered $1 \mathrm{~h}$ after unilateral carotid ligation (Fig. 5F). P7 pups that received VU after

242 1h of ischemic seizures developed a significant aggravation of EEG seizure burden in the

243 second hour (Fig. 5F-J). Taken together, KCC2 inhibition induced epileptiform activity in

244 the naïve neonatal brain and exacerbated ischemic neonatal seizures at P7.

246 In vitro CLP257 incubation increases KCC2 membrane insertion

247 The KCC2 functional enhancer CLP257 has been shown to increase chloride

248 extrusion capacity and KCC2 membrane expression in vitro, and it has been suggested

249 that the net effect of KCC2 functional enhancement may emerge from relatively small

250 changes in KCC2 function (11, 20, 37). The neonatal brain tightly regulates KCC2 activity

251 via S940 and T1007 phosphorylation (23), and it is unknown if age-dependent 
252 mechanisms affect KCC2 functional enhancement. Therefore, P7 neonatal brain sections

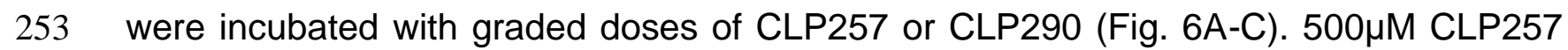

254 significantly increased membrane KCC2 and S940 expression (Fig. 6B). The

255 phosphorylation of S940 increases KCC2 plasma membrane accumulation and transport

256 activity $(38,39)$. To assess if the S940 site was necessary for CLP257 mediated KCC2

257 membrane insertion, brain sections from S940A ${ }^{+/+}$knock-in mutant mice (25) were treated

258 with CLP257 (Fig. 6 D-G). Without the ability to phosphorylate S940, CLP257 failed to

259 increase KCC2 membrane insertion (Fig. 6 F).

Ischemic neonatal seizures do not modulate KCC2-T1007 phosphorylation

The phosphorylation of KCC2 residue T1007 inhibits KCC2 function $(40,41)$. It has

263 been proposed that KCC2 functional enhancers must either increase KCC2 surface

264 stability and/or decrease T1007 phosphorylation (42, 43). Therefore, T1007

265 phosphorylation was investigated $24 \mathrm{~h}$ after $\mathrm{P} 7$ ischemic neonatal seizures (Fig. 7A-C).

266 Ischemia did not significantly change T1007 expression at $24 \mathrm{~h}$ in either hemisphere (Fig.

267 7A-C and Supplemental Fig. 4). The effective CLP290 10' dose also did not significantly

268 modulate T1007 expression, however bolus administration of $20 \mathrm{mg} / \mathrm{kg}$ CLP290 resulted

269 in a significant increase in T1007 at 24h in both pre and post treatment groups (Fig. 7A-

270 D). To investigate if pharmacomodulation of KCC2 is governed by T1007, naïve CD-1 P7

271 brain sections were treated with CLP257 (Fig. 7E-H). All groups treated with CLP257

272 showed lower T1007 expression at the membrane than untreated slices (Fig. 7G)

273 supporting the data from the in vivo experiments (Fig. 7B \&C). 
275 Spontaneous epileptiform discharges in $5940 A^{+/+}$pups

276 KCC2 deficient mice die postnatally with generalized seizures and respiratory failure

$277(44,45)$. S940A ${ }^{+/+}$mice are susceptible to death after kainate induced status epilepticus

278 in adulthood (36). In patients with idiopathic generalized epilepsy and early childhood

279 onset of febrile seizures, heterozygous missense variants in SLC12A5 have been

280 identified $(46,47)$ and associated with a reduction in $S 940$ phosphorylation (46).

281 However, it is unknown if the prevention of $\$ 940$ phosphorylation alone can induce

282 spontaneous epileptiform activity during the neonatal period. Therefore, S940A ${ }^{+/+}$pups

283 underwent vEEG at P7 and P12 (Fig. 8A-D). S940A+/+ mice had spontaneous epileptiform

284 discharges at both P7 and P12, which failed to respond to CLP290 intervention (Fig.

285 8AD). These results suggest that the prevention of S940 phosphorylation is sufficient for 286 spontaneous epileptic activity during development.

$288 T_{1007 A^{+/+}}$pups are resistant to ischemic seizures

289 T1007 phosphorylation decreases during development and is associated with an

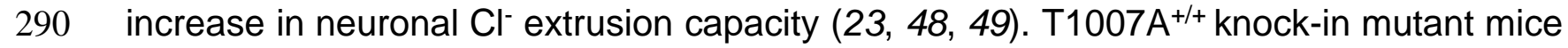

291 have a reduced susceptibility to kainate induced status epilepticus in adulthood (24). It is

292 unknown if KCC2 phosphorylation alone can modulate the susceptibility to ischemic 293 neonatal seizures. To investigate the role of KCC2 phosphorylation in ischemic neonatal 294 seizures WT, S940A+/+, and T1007A+/+ P7 pups underwent unilateral carotid ligation with 295 2h vEEG recordings (Fig. 8E-G). After P7 unilateral carotid ligation T1007A+/+ pups were 296 significantly resistant to ischemic seizures than WT (Fig. 8E-G). This result suggests that 297 reducing T1007 phosphorylation on KCC2 is a promising therapeutic target to be 
298 developed for neonatal seizures. S940A ${ }^{+/+}$pups had higher seizure burdens than WT after

299 ischemia (Fig. 8G). CLP290 10' treatment did not improve the efficacy of PB in S940A ${ }^{+/+}$

300 mice but significantly increased $1^{\text {st }}$ hour seizure burden when compared to untreated

301 S940A $^{+/+}$mice (Fig. 8H). During development, mice undergo a reduction in ischemic

302 seizure susceptibility from P7 to P10 that is associated with a robust upregulation of KCC2

303 and $\mathrm{S} 940$ phosphorylation $(15,26)$. S940A ${ }^{+/+}$mice did not undergo a developmental

304 decrease in ischemic seizure susceptibility at P10 when compared to P7 S940A ${ }^{+/+}$mice $^{-1}$

305 (Fig. 8I; P=0.57). These results suggest that both the CLP290-mediated KCC2 functional

306 enhancement and the age-dependent reduction in ischemic seizure susceptibility are

307 dependent upon S940 phosphorylation.

308

S940A+/+ pups are susceptible to status epilepticus and death

To determine if the ability for KCC2 phosphorylation to modulate seizure

311 susceptibility was specific to ischemic seizures, WT, S940A+/+, and T1007A ${ }^{+/+}$P12 pups

312 underwent the 3h PTZ challenge (Fig. 8J-O). As described previously (Fig. 4), pups were

313 administered three doses of PTZ $(20,20$, and $40 \mathrm{mg} / \mathrm{kg})$ 1h apart. S940A ${ }^{+/+}$mice

314 immediately progressed into status epilepticus and died before the $2^{\text {nd }} \mathrm{h}$ (Fig. $8 \mathrm{~J}-\mathrm{O}$ ). This

315 indicated that the prevention of $\mathrm{S} 940$ phosphorylation increased the risk of sudden

316 unexpected death in epilepsy (SUDEP)-like phenomenon (50). In contrast, T1007A ${ }^{+/+}$

317 mice were resistant to the PTZ induced seizures when compared to WT (Fig. 8 M).

318 Additionally, the assessment of righting reflex as a neurodevelopmental milestone

319 identified that only S940A $\mathrm{A}^{+/+}$mice had a significantly impaired righting reflex at P7 but not 
320 at P12 (Fig. 8P). These data suggest that KCC2 phosphorylation controls seizure

321 susceptibility during development.

322

323 Materials and Methods

324 Experimental Paradigm

325 In CLP290 and CLP257 experiments, all pups regardless of treatment group 326 received a loading dose of PB $(25 \mathrm{mg} / \mathrm{kg})$ dissolved in $100 \%$ isotonic phosphate-buffered 327 saline (PBS) delivered via intraperitoneal (IP) injection at $1 \mathrm{~h}$. All injections regardless of 328 the treatment group, drug, or age were administered using Hamilton syringes. All drugs 329 were prepared the day of experiments. CLP290 and CLP257 were both dissolved in 330 45/55\% 2-Hydroxypropyl- $\beta$-cyclodextrin (HPCD)/PBS with a $\mathrm{pH}$ range between 7.2-7.5. To assess the efficacy of CLP290 treatment at P7, pups were assigned to the PB-

332 only, post, primed, or pre-treatment groups (Fig. 1). PB-only treatment was defined by the 333 single administration of PB at $1 \mathrm{~h}$ without further intervention. Post treatment was denoted 334 by administration of CLP290 immediately following unilateral carotid ligation with PB at 335 1h. 5, 10, or $20 \mathrm{mg} / \mathrm{kg}$ CLP290 was administered via IP injections to P7 pups in the post 336 treatment groups (i.e. P7 CLP290 Post 5, 10, and 20). The primed treatment group was

337 defined by the administration of CLP290 4h preceding unilateral carotid ligation and 338 another dose immediately following unilateral carotid ligation with PB at $1 \mathrm{~h}$. The 339 pretreatment group was denoted by the single administration of CLP290 4h before 340 unilateral carotid ligation with PB at 1h after ligation (i.e. P7 CLP290 Pre 20").

341 As a carbamate prodrug of CLP257, CLP290 has an improved bioavailability in 342 P7 CD-1 pups when compared to CLP257. To investigate the differential effects of 
343 CLP290 and CLP257 on neonatal seizure suppression, P7 pups were administered

$34410 \mathrm{mg} / \mathrm{kg}$ CLP257 in a primed-treatment group with PB at $1 \mathrm{~h}$ after ligation. At P10, when

345 seizures after unilateral carotid ligation are responsive to $P B$, the efficacy of $10 \mathrm{mg} / \mathrm{kg}$

346 CLP290 to improve seizure suppression was investigated. P10 pups were either assigned

347 to the post, primed, or pretreatment groups with PB $1 \mathrm{~h}$ after ligation.

349 Animals

All experimental procedures and protocols were conducted in compliance with 351 guidelines by the Committee on the Ethics of Animal Experiments (Permit Number: 352 A3272-01) and were approved by the Animal Care and Use of Committee of Johns 353 Hopkins University. CD1 litters were purchased from Charles River Laboratories 354 (Wilmington, MA.). Newly born CD-1 litters $(n=10)$ were delivered with a dam at postnatal 355 day three or four and allowed to acclimate. S940A ${ }^{+/+}$and T906A/T1007A ${ }^{+/+}$mice were a 356 gift from Stephen J. Moss Laboratories at the Tufts University School of Medicine.

357 Equivalent numbers of male and female pups were introduced into the study. All mice 358 were housed on a 12h light-dark cycle with food and water provided ad libitum. For 359 surgical procedures and western blotting see Supplement.

\section{In vivo video-EEG recording and analyses}

EEG recordings were acquired using Sirenia Acquisition software with 363 synchronous video capture (Pinnacle Technology Inc. KS, USA). Data acquisition was 364 done with sampling rates of $400 \mathrm{~Hz}$ that had a preamplifier gain of 100 and the filters of $3650.5 \mathrm{~Hz}$ high-pass and $50 \mathrm{~Hz}$ low-pass. The data were scored by binning EEG in 10 s 
366 epochs. Similar to our previous studies(15), seizures were defined as electrographic ictal

367 events that consisted of rhythmic spikes of high amplitude, diffuse peak frequency of $\geq 7$ -

$3688 \mathrm{~Hz}$ (i.e.; peak frequency detected by automated spectral power analysis) lasting $\geq 6 \mathrm{~s}$

369 (i.e.; longer than half of each 10 s epoch). Short duration burst activity lasting $<6$ s (brief

370 runs of epileptiform discharges) was not included for seizure burden calculations similar

371 to previous studies in the model. Mean time spent seizing for $1^{\text {sth }}$ baseline seizure burden

372 vs. $2^{\text {ndh }}$ post-PB seizure burden was quantified in seconds. Mean seizure suppression

373 was calculated using Equation 1:

$$
\% \text { seizure suppression }=\frac{\left(1^{\text {st }} h \text { seizure burden }-2^{\text {nd }} h \text { seizure burden }\right)}{\left(1^{\text {st }} h \text { seizure burden }\right)} * 100
$$

Mean ictal events and ictal durations (seconds/event) were calculated for $1^{\text {sth }}$ vs.

$3762^{\text {nd }} \mathrm{h}$.

Statistics

For all experiments, the quantification and analysis of data were performed blinded

to the genotype, sex, and treatment conditions. All statistical tests were performed using

381 GraphPad Prism software. Two-way analysis of variance (ANOVA) was performed with

382 Tukey's post hoc correction. One-way ANOVA was performed with Dunnett's post hoc

383 correction. Paired and unpaired t-tests were two-tailed. Survival analysis was performed

384 by a Mantel-Cox test. Data are represented as bar graphs representing the mean, with

385 dot plots representing each individual data point. Errors bars are \pm 1 standard error of

386 mean. $P$ values for $\leq 0.05$ are reported. 


\section{Discussion}

$\mathrm{KCC} 2$ is one of the key regulators of intracellular chloride $(7,51)$. However, diverse

391 mechanisms regulate $\mathrm{KCC} 2$ membrane insertion and $\mathrm{Cl}^{-}$extrusion capacity $(11,12)$.

392 Modulating KCC2 function to enhance inhibition has become a focused area of research

393 to help identify therapeutic targets for pathologies with documented KCC2 degradation or

394 hypofunction. Enhancement of KCC2 membrane stability and function could reestablish

395 synaptic inhibition in seizing neonatal brains when positive GABAR modulators like PB

396 fail to curb severe recurrent seizures. The goal would be to acutely rescue KCC2 function

397 by preventing further degradation and maintaining KCC2 membrane stability. For

398 translational applications, this strategy would target pathologies with documented KCC2

399 hypofunction such as recent reports of significant reduction in KCC2-positive cells in

400 postmortem cerebral samples from preterm infants with white matter injury (52). This

401 strategy is distinct from overexpression or enhancement of KCC2 function in neurons with

402 stable endogenous KCC2 expression which could be detrimental to developing brains

403 (53,54). Moderate and severe HIE seizures are clinically associated with significant

404 hourly/daily seizure burdens which tend to cluster into high-seizing and non-seizing

405 periods $(4,55)$, however they are transient in nature. This clustering phenotype makes

406 continuous EEG monitoring during the acute period a necessity and gold standard (56,

40757 ) to determine both the true severity of the seizures and the efficacy of ASM

408 interventions. An acute protocol of rescuing KCC2 hypofunction during this critical period

409 would help mitigate both the refractory neonatal seizures and their long-term

410 consequences. 
For this reason we tested acute intervention protocols for multiple graded doses of

412 the KCC2 functional enhancer CLP290 both as post and primed dosing in our CD-1

413 mouse model of refractory neonatal seizures. Our seminal results show that CLP290 is

414 significantly efficacious in reversing PB-resistant seizures at P7 in a dose-dependent

415 manner that plateaus at the highest dose tested in this study. The ability of CLP290 to

416 rescue both $\mathrm{KCC} 2$ expression and $-\mathrm{S} 940$ phosphorylation at $24 \mathrm{~h}$ post-ischemia were

417 similar to those reported with the TrkB antagonist ANA12 but also distinct since CLP290

418 had no effect on subduing the ischemia-induced TrkB activation (17). Further its ability to

419 significantly increase KCC2 membrane insertion in P7 brain sections and inability subdue

420 ischemic seizures in the mutant $\mathrm{KCC} \mathrm{S940 \textrm {A } ^ { + / + }}$ pups support its role as a KCC2

421 functional enhancer acting through its S940 phosphorylation site.

\section{Novel anti-seizure protocols that help mitigate epileptogenesis}

424 The long-term effects of refractory seizures and ASM interventions are major

425 factors to consider for neonatal antiseizure management $(21,22)$. It has been shown that 426 chemoconvulsant induced seizures upregulate KCC2 expression $(32,58)$. Therefore, an

427 initial low-dose PTZ would be expected to upregulate KCC2 in P12 naïve pups likely

428 making them resistant to additional seizures. The proof of concept data reported here in

429 the naïve pups for this novel assay supported this reasoning. The failure of the initial sub-

430 threshold PTZ dose to confer this resistance in the PB-only group indicated a heightened

431 susceptibility to epileptogenesis at P12. In contrast, the P7 CLP290 treated pups showed

432 significant protection from this epileptogenesis. Neonatal ischemia results in $\sim 20-40 \%$

433 decrease in KCC2 expression which recovers endogenously over the next few days and 
434 catches up to its developmental trajectory (15). The ability to rescue KCC2 hypofunction

435 acutely during this period could help mitigate the onset of long-term impairments in circuit

436 function opening up a promising window for transient therapy aimed at rescuing KCC2

437 hypofunction.

KCC2 hypofunction can independently initiate and aggravate neonatal seizures

441 of the selective KCC2 inhibitor VU was tested in naïve CD-1 pups at P7. During

442 development KCC2 undergoes a significant increase in expression (6) associated with a

443 phosphorylation profile that coincides with a maturational increase in $\mathrm{Cl}$ - extrusion 444 capacity (23). The dose-dependent emergence of spontaneous epileptiform events with 445 graded severity in the naïve P7 CD-1 pups highlighted the significant role of KCC2 446 function in preventing ictogenesis in the immature brain (59). KCC2 antagonism during 447 ischemic seizures further aggravated seizure severity at P7, supporting its role in the 448 severity of seizure burdens. The immature brain has an inherent susceptibility to 449 excitotoxic injury with propensity for seizures $(1,59)$. Our data indicate that KCC2 function 450 in the neonatal brain plays a significant role in maintaining the balance between excitation 451 and inhibition.

\section{Novel targets to rescue KCC2 hypofunction are needed}

454 Currently there are very few studies evaluating CLP290 efficacy in models of 455 neurological disease and there is an urgent need for the discovery of additional novel 456 KCC2 functional enhancers. The De Koninck group originally identified CLP257 as a 
457 KCC2 functional enhancer using high throughput screening (20). The prodrug CLP290

458 was shown to have improved pharmacokinetics over CLP257 and rescued neuropathic

459 pain in a rat model. In vitro studies proposed that the active drug CLP257 does not directly

460 modulate KCC2 activity but potentiates $\operatorname{GABA}_{A} R$ activity $(60,61)$. Regardless, CLP290

461 has been shown to improve outcomes in models of neuropathic pain and spinal cord injury

462 associated with KCC2 hypofunction $(20,62,63)$. The results of this current study highlight

463 the significant role of KCC2 and its phosphorylation sites in neonatal seizure severity and

464 mechanisms underlying CLP290 ASM efficacy using both in vivo and in vitro protocols.

465 The identification of newer and more efficient KCC2 functional enhancers targeting these

466 phosphorylation sites are needed.

467 The mechanisms by which CLP290 enhances KCC2 function in neurons are not 468 well understood $(20,60,61)$. We investigated the role of the known phosphorylation sites

469 on KCC2 those that either positively or negatively regulate KCC2 membrane stability and

$470 \mathrm{Cl}^{-}$extrusion capacity, in the two mutant mice. The $5940 \mathrm{~A}^{+/+}$pups not only showed an

471 increase in seizure susceptibility to the P7 ischemic insult, but importantly we documented

472 the occurrence of spontaneous epileptiform discharges in the naïve mutant pups at P7.

$473 \mathrm{~S}_{40 \mathrm{~A}^{+/+}}$pups did not show the age-dependent (P7 vs. P10) differences in ischemic

474 seizure susceptibility highlighting the developmental role of S940 phosphorylation in

475 hyperexcitability of the immature brain. The significance of this finding is highlighted in

476 recent reports of KCC2 mutations in early-onset epileptic syndromes (9), in which mutant

$477 \mathrm{KCC} 2$ is associated with a reduction in S940 phosphorylation (46) and impaired $\mathrm{Cl}^{-}$

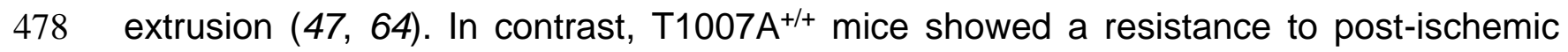

479 seizure susceptibility as compared to their WT littermates following P7 ischemic insults. 
480 Additionally, when the repeated-dose PTZ protocol was tested in both mutant strains at

$481 \mathrm{P} 12$, the high seizure burdens and early mortality detected in S940A+/+ pups and low 482 seizure susceptibility of the $\mathrm{T}_{1007 \mathrm{~A}^{+/+}}$pups may further support the role of the two sites 483 in the evolution of epileptogenesis following P7 neonatal seizures in the CD-1 model.

485 Activation of KCC2 homeostatic compensatory mechanisms with bolus doses of CLP290

In contrast to the graded ASM responses of CLP290 for the lower doses tested, the anti-seizure responses identified for the highest CLP290 dose $(20 \mathrm{mg} / \mathrm{kg}$; both Pre and Post) tested in this study showed differences. First hour baseline seizure burdens which remained similar to ligate-only group for the lower doses of CLP290 were

491 significantly suppressed with the post-ligation bolus dose of $20 \mathrm{mg} / \mathrm{kg}$ (Suppl Fig 1 ). This

492 alteration of the baseline seizure susceptibility at P7 with $20 \mathrm{mg} / \mathrm{kg}$ of CLP290 took away 493 the graded nature on the reversal of PB-refractoriness seen with the $5 \mathrm{vs} .10 \mathrm{mg} / \mathrm{kg}$ doses 494 (Fig.1).

KCC2 can autoregulate its $\mathrm{Cl}^{-}$extrusion capacity using positive and negative modulators that activate its multiple phosphorylation sites (23) .It is also known that

497 modest KCC2 hypofunction can play a significant role in the emergence of neurological 498 disorders (37). In contrast, overexpression of KCC2 in neurons could not only be 499 detrimental but also trigger endogenous homeostatic pathways for the negative regulation 500 of KCC2 function. Interestingly, pushing KCC2 function beyond its physiological levels 501 was found to be difficult in simulation studies (11). The high bolus dose of CLP290 tested 502 here was found to activate one such endogenous mechanism by which KCC2 
503 upregulation as denoted by a significant change in ischemic seizure susceptibility at P7

504 was homeostatically countered by a significant upregulation of T1007 phosphorylation at

$50524 \mathrm{~h}$. The upregulation of T1007 in the CLP290 20mg/kg treatment group was not detected

506 in the untreated P7 CD-1 ischemia group nor the with the lower doses of CLP290 and

507 therefore may indicate a homeostatic regulation of KCC2 function induced by CLP290

508 high bolus dose at $24 \mathrm{~h}$ that was independent of the initial ischemic insult. The findings of

509 the CLP257-mediated T007 phosphorylation in vitro versus in vivo experiments indicate

510 a temporal regulation of the homeostatic response with T1007 upregulation which needs

511 further investigation.

513 Novel insights for the next generation of the next generation of KCC2 enhancers

514 Recent studies have shown that during induced status epilepticus, reduced $\mathrm{Cl}^{-}$

515 extrusion capacity and exacerbated activity-dependent $\mathrm{Cl}^{-}$loading can result in

516 GABAergic transmission being ictogenic (14). Optogenetic stimulation of GABAergic

517 interneurons in this status epilepticus-like state enhanced the epileptiform activity in a

$518 \mathrm{GABA}_{A} \mathrm{R}$ dependent manner (65) indicating GABA-mediated depolarization. These

519 findings support data both from our model and clinical reports where the when initial

520 loading-dose of PB fails to curb seizures, additional PB doses do not help rescue the

521 refractoriness $(2,66)$. Additionally, given the toxicity of high-dose PB on neonatal brains

522 such protocols may be counterproductive in the short and long-term (67-69).

KCC2 hypofunction is emerging as a significant cause underlying impaired

524 inhibition in multiple neurological disorders $(5,52,65)$. Interestingly, the research into

525 both refractory seizures and refractory spinal nerve pain has identified KCC2 
526 hypofunction as a common underlying cause. Here we have shown that enhancing KCC2

527 function in a well-characterized preclinical model of refractory seizures can rescue not

528 only the acute PB-refractoriness but also help mitigate epileptogenesis with early

529 intervention. Although additional studies are needed to investigate the direct and indirect

530 effects of enhancing $\mathrm{Cl}^{-}$extrusion capacity of $\mathrm{KCC} 2$ in the immature brain, the novel

531 findings reported here highlight the role of KCC2 hypofunction and its phosphorylation

532 sites in HIE-related refractory seizures and an evidence based focus on targeting KCC2

533 function in development of future translational strategies.

540 Acknowledgements: We thank Professor Stephen J. Moss (Tufts University School of

541 Medicine) for the generous transfer of the S940A+/+ and T1007A ${ }^{+/+}$mice (MTA). We thank

542 Dr. Tarek Deeb (Tufts University School of Medicine) and Dr. Yvonne Moore (Tufts

543 University School of Medicine) for useful discussions and technical advice. We thank

544 Professor Yves De Koninck (Université Laval) for generously sharing aliquots of CLP290

545 (MTA). We thank Dr. Rana Rais from the Johns Hopkins Drug Discovery Core for the

546 HPLC experiments. 


\section{Funding}

550 Research reported in the publication was supported by the Eunice Kennedy Shriver

551 National Institute of Child Health and Human Development of National Institutes of Health

552 under Grant No. R01HD090884 (SDK). The content is solely the responsibility of the 553 authors and does not necessarily represent the official view of the NIH.

\section{Author Contributions}

556 SDK conceived the project. BJS, PAK, BMC, and SDK acquired data. BJS, PAK, and 557 SDK analyzed data. BJS, PAK, and SDK wrote the paper. All authors have seen and 558 approved the manuscript, and this manuscript has not been published elsewhere.

$561 \quad$ Competing Interests

562 SDK is listed as an author on US patent 10525024B2, "Methods for rescuing 563 phenobarbital resistance of seizures by ANA-12 or ANA-12 in combination with CLP290." 564 


\section{References}

573

574

575

576

577

578

579

580

581

582

583

584

585

586

587

588

589

590

591

592

593

594

595

596

597

598

599

600

601

602

603

604

1. J. Volpe, T. Inder, B. Darras, L. de Vries, A. du Plessis, J. Neill, J. Perlman, Volpe's Neurology of the Newborn - 6th Edition (Elsevier, ed. 6th, 2017).

2. R. Sankar, M. J. Painter, Neonatal seizures: after all these years we still love what doesn't work. Neurology. 64, 776-777 (2005).

3. H. C. Glass, J. S. Soul, C. J. Chu, S. L. Massey, C. J. Wusthoff, T. Chang, M. R. Cilio, S. L. Bonifacio, N. S. Abend, C. Thomas, M. Lemmon, C. E. McCulloch, R. A. Shellhaas, Response to antiseizure medications in neonates with acute symptomatic seizures. Epilepsia. 60, e20-e24 (2019).

4. G. B. Boylan, N. J. Stevenson, S. Vanhatalo, Monitoring neonatal seizures. Semin. Neonatal Med. 18, 202-208 (2013).

5. K. Kaila, T. J. Price, J. A. Payne, M. Puskarjov, J. Voipio, Cation-chloride cotransporters in neuronal development, plasticity and disease. Nat Rev Neurosci. 15, 637-654 (2014).

6. G. Sedmak, N. Jovanov-Milošević, M. Puskarjov, M. Ulamec, B. Krušlin, K. Kaila, M. Judaš, Developmental Expression Patterns of KCC2 and Functionally Associated Molecules in the Human Brain. Cereb. Cortex. 26, 4574-4589 (2016).

7. K. Kaila, T. J. Price, J. A. Payne, M. Puskarjov, J. Voipio, Cation-chloride cotransporters in neuronal development, plasticity and disease. Nat. Rev. Neurosci. 15, 637-654 (2014).

8. Y. Ben-Ari, Excitatory actions of GABA during development: the nature of the nurture. Nat Rev Neurosci. 3, 728-739 (2002).

9. P. Q. Duy, W. B. David, K. T. Kahle, Identification of KCC2 Mutations in Human Epilepsy Suggests Strategies for Therapeutic Transporter Modulation. Front. Cell. Neurosci. 13 (2019), doi:10.3389/fncel.2019.00515.

10. French Jacqueline A., Refractory Epilepsy: Clinical Overview. Epilepsia. 48, 3-7 (2007).

11. N. Doyon, L. Vinay, S. A. Prescott, Y. De Koninck, Chloride Regulation: A Dynamic Equilibrium Crucial for Synaptic Inhibition. Neuron. 89, 1157-1172 (2016).

12. B. J. Sullivan, S. D. Kadam, in Neuronal Chloride Transporters in Health and Disease, X. Tang, Ed. (Elsevier, ed. 1, 2020), p. 650.

13. R. Nardou, S. Yamamoto, G. Chazal, A. Bhar, N. Ferrand, O. Dulac, Y. Ben-Ari, I. Khalilov, Neuronal chloride accumulation and excitatory GABA underlie aggravation of neonatal epileptiform activities by phenobarbital. Brain J. Neurol. 134, 987-1002 (2011).

14. R. J. Burman, J. S. Selfe, J. H. Lee, M. van den Berg, A. Calin, N. K. Codadu, R. Wright, S. E. Newey, R. R. Parrish, A. A. Katz, J. M. Wilmshurst, C. J. Akerman, A. J. Trevelyan, J. 
V. Raimondo, Excitatory GABAergic signalling is associated with benzodiazepine resistance in status epilepticus. Brain J. Neurol. 142, 3482-3501 (2019).

15. S. K. Kang, G. J. Markowitz, S. T. Kim, M. V. Johnston, S. D. Kadam, Age- and sexdependent susceptibility to phenobarbital-resistant neonatal seizures: role of chloride cotransporters. Front Cell Neurosci. 9, 173- (2015).

16. S. K. Kang, M. V. Johnston, S. D. Kadam, Acute TrkB inhibition rescues phenobarbitalresistant seizures in a mouse model of neonatal ischemia. Eur. J. Neurosci. 42, 2792-2804 (2015).

17. B. M. Carter, B. J. Sullivan, J. R. Landers, S. D. Kadam, Dose-dependent reversal of KCC2 hypofunction and phenobarbital-resistant neonatal seizures by ANA12. Sci. Rep. 8, 11987

18. S. K. Kang, S. Ammanuel, S. Thodupunuri, D. A. Adler, M. V. Johnston, S. D. Kadam, Sleep dysfunction following neonatal ischemic seizures are differential by neonatal age of insult as determined by qEEG in a mouse model. Neurobiol. Dis. 116, 1-12 (2018).

19. S. K. Kang, S. Ammanuel, D. A. Adler, S. D. Kadam, Rescue of PB-resistant neonatal seizures with single-dose of small-molecule TrkB antagonist show long-term benefits. Epilepsy Res. 159, 106249 (2020).

20. M. Gagnon, M. J. Bergeron, G. Lavertu, A. Castonguay, S. Tripathy, R. P. Bonin, J. PerezSanchez, D. Boudreau, B. Wang, L. Dumas, I. Valade, K. Bachand, M. Jacob-Wagner, C. Tardif, I. Kianicka, P. Isenring, G. Attardo, J. A. M. Coull, Y. De Koninck, Chloride extrusion enhancers as novel therapeutics for neurological diseases. Nat. Med. 19, 15241528 (2013).

21. H. C. Glass, Z. M. Grinspan, R. A. Shellhaas, Outcomes after acute symptomatic seizures in neonates. Semin. Fetal. Neonatal Med. 23, 218-222 (2018).

22. S. K. Kang, S. D. Kadam, Neonatal Seizures: Impact on Neurodevelopmental Outcomes. Front Pediatr. 3, 101- (2015).

23. Y. E. Moore, L. C. Conway, N. J. Brandon, T. Z. Deeb, S. J. Moss, Developmental regulation of KCC2 phosphorylation has long-term impacts on cognitive function. Front. Mol. Neurosci. 12 (2019), doi:10.3389/fnmol.2019.00173.

24. Y. E. Moore, T. Z. Deeb, H. Chadchankar, N. J. Brandon, S. J. Moss, Potentiating KCC2 activity is sufficient to limit the onset and severity of seizures. Proc. Natl. Acad. Sci. 115, 10166-10171 (2018). Brandon, J. Dunlop, J. Maguire, P. A. Davies, S. J. Moss, KCC2 activity is critical in limiting the onset and severity of status epilepticus. Proc. Natl. Acad. Sci. U. S. A. 112, 3523-3528 (2015). 
26. P. A. Kipnis, B. J. Sullivan, B. M. Carter, S. D. Kadam, TrkB agonists prevent postischemic emergence of refractory neonatal seizures in mice. JCI Insight. 5 (2020), doi:10.1172/jci.insight.136007.

27. S. C. Kharod, S. K. Kang, S. D. Kadam, Off-label use of bumetanide for brain disorders: An overview. Front. Neurosci. 13 (2019), doi:10.3389/fnins.2019.00310.

28. M. Puskarjov, K. T. Kahle, E. Ruusuvuori, K. Kaila, Pharmacotherapeutic targeting of cation-chloride cotransporters in neonatal seizures. Epilepsia. 55, 806-818 (2014).

29. C. P. Panayiotopoulos, Neonatal Seizures and Neonatal Syndromes (Bladon Medical Publishing, 2005; https://www.ncbi.nlm.nih.gov/books/NBK2599/).

30. E. Kossoff, Neonatal Seizures Due to Hypoxic-Ischemic Encephalopathy: Should We Care? Epilepsy Curr. 11, 147-148 (2011).

31. S. K. Kang, S. Ammanuel, S. Thodupunuri, D. A. Adler, M. V. Johnston, S. D. Kadam, Sleep dysfunction following neonatal ischemic seizures are differential by neonatal age of insult as determined by qEEG in a mouse model. Neurobiol. Dis. 116, 1-12 (2018).

32. S. C. Kharod, B. M. Carter, S. D. Kadam, Pharmaco-resistant neonatal seizures: critical mechanistic insights from a chemoconvulsant model. Dev. Neurobiol. (2018), doi:10.1002/dneu.22634.

33. S. Sivakumaran, R. A. Cardarelli, J. Maguire, M. R. Kelley, L. Silayeva, D. H. Morrow, J. Mukherjee, Y. E. Moore, R. J. Mather, M. E. Duggan, N. J. Brandon, J. Dunlop, S. Zicha, S. J. Moss, T. Z. Deeb, Selective Inhibition of KCC2 Leads to Hyperexcitability and Epileptiform Discharges in Hippocampal Slices and In Vivo. J. Neurosci. 35, 8291-8296 (2015).

34. C. Rivera, J. Voipio, J. Thomas-Crusells, H. Li, Z. Emri, S. Sipilä, J. A. Payne, L. Minichiello, M. Saarma, K. Kaila, Mechanism of Activity-Dependent Downregulation of the Neuron-Specific K-Cl Cotransporter KCC2. J. Neurosci. 24, 4683-4691 (2004).

35. H. R. Pathak, F. Weissinger, M. Terunuma, G. C. Carlson, F.-C. Hsu, S. J. Moss, D. A. Coulter, Disrupted Dentate Granule Cell Chloride Regulation Enhances Synaptic Excitability during Development of Temporal Lobe Epilepsy. J. Neurosci. 27, 1401214022 (2007).

36. L. Silayeva, T. Z. Deeb, R. M. Hines, M. R. Kelley, M. B. Munoz, H. H. C. Lee, N. J. Brandon, J. Dunlop, J. Maguire, P. A. Davies, S. J. Moss, KCC2 activity is critical in limiting the onset and severity of status epilepticus. Proc. Natl. Acad. Sci. U. S. A. 112, 3523-3528 (2015).

37. N. Doyon, S. A. Prescott, Y. De Koninck, Mild KCC2 Hypofunction Causes Inconspicuous Chloride Dysregulation that Degrades Neural Coding. Front. Cell. Neurosci. 9 (2016), doi:10.3389/fncel.2015.00516. 
38. H. H. C. Lee, J. A. Walker, J. R. Williams, R. J. Goodier, J. A. Payne, S. J. Moss, Direct Protein Kinase C-dependent Phosphorylation Regulates the Cell Surface Stability and Activity of the Potassium Chloride Cotransporter KCC2. J. Biol. Chem. 282, 29777-29784 (2007).

39. H. H. Lee, T. Z. Deeb, J. A. Walker, P. A. Davies, S. J. Moss, NMDA receptor activity downregulates $\mathrm{KCC} 2$ resulting in depolarizing $\mathrm{GABA}(\mathrm{A})$ receptor mediated currents. Nat. Neurosci. 14, 736-743 (2011).

40. J. Rinehart, Y. D. Maksimova, J. E. Tanis, K. L. Stone, C. A. Hodson, J. Zhang, M. Risinger, W. Pan, D. Wu, C. M. Colangelo, B. Forbush, C. H. Joiner, E. E. Gulcicek, P. G. Gallagher, R. P. Lifton, Sites of regulated phosphorylation that control K-Cl cotransporter activity. Cell. 138, 525-536 (2009).

41. S. Titz, E. M. Sammler, S. G. Hormuzdi, Could tuning of the inhibitory tone involve graded changes in neuronal chloride transport? Neuropharmacology. 95, 321-331 (2015).

42. L. C. Conway, R. A. Cardarelli, Y. E. Moore, K. Jones, L. J. McWilliams, D. J. Baker, M. P. Burnham, R. W. Bürli, Q. Wang, N. J. Brandon, S. J. Moss, T. Z. Deeb, N-Ethylmaleimide increases KCC2 cotransporter activity by modulating transporter phosphorylation. J. Biol. Chem. 292, 21253-21263 (2017).

43. Y. E. Moore, M. R. Kelley, N. J. Brandon, T. Z. Deeb, S. J. Moss, Seizing Control of KCC2: A New Therapeutic Target for Epilepsy. Trends Neurosci. 40, 555-571 (2017).

44. N.-S. Woo, J. Lu, R. England, R. McClellan, S. Dufour, D. B. Mount, A. Y. Deutch, D. M. Lovinger, E. Delpire, Hyperexcitability and epilepsy associated with disruption of the mouse neuronal-specific K-Cl cotransporter gene. Hippocampus. 12, 258-268 (2002).

45. C. A. Hubner, Disruption of $\mathrm{KCC} 2$ reveals an essential role of $\mathrm{K}-\mathrm{Cl}$ cotransport already in early synaptic inhibition. Neuron. 30, 515-524 (2001).

46. K. T. Kahle, N. D. Merner, P. Friedel, L. Silayeva, B. Liang, A. Khanna, Y. Shang, P. Lachance-Touchette, C. Bourassa, A. Levert, P. A. Dion, B. Walcott, D. Spiegelman, A. Dionne-Laporte, A. Hodgkinson, P. Awadalla, H. Nikbakht, J. Majewski, P. Cossette, T. Z. Deeb, S. J. Moss, I. Medina, G. A. Rouleau, Genetically encoded impairment of neuronal $\mathrm{KCC} 2$ cotransporter function in human idiopathic generalized epilepsy. EMBO Rep. 15, 766-774 (2014).

47. M. Puskarjov, P. Seja, S. E. Heron, T. C. Williams, F. Ahmad, X. Iona, K. L. Oliver, B. E. Grinton, L. Vutskits, I. E. Scheffer, S. Petrou, P. Blaesse, L. M. Dibbens, S. F. Berkovic, K. Kaila, A variant of KCC2 from patients with febrile seizures impairs neuronal Clextrusion and dendritic spine formation. EMBO Rep. 15, 723-729 (2014).

48. L. I. Pisella, J.-L. Gaiarsa, D. Diabira, J. Zhang, I. Khalilov, J. Duan, K. T. Kahle, I. Medina, Impaired regulation of KCC2 phosphorylation leads to neuronal network dysfunction and neurodevelopmental pathology. Sci. Signal. 12 (2019), doi:10.1126/scisignal.aay0300. 
49. M. Watanabe, J. Zhang, M. S. Mansuri, J. Duan, J. K. Karimy, E. Delpire, S. L. Alper, R. P. Lifton, A. Fukuda, K. T. Kahle, Developmentally regulated KCC2 phosphorylation is essential for dynamic GABA-mediated inhibition and survival. Sci. Signal. 12 (2019), doi:10.1126/scisignal.aaw9315.

50. L. Nashef, E. L. So, P. Ryvlin, T. Tomson, Unifying the definitions of sudden unexpected death in epilepsy. Epilepsia. 53, 227-233 (2012).

51. G. Gamba, Molecular Physiology and Pathophysiology of Electroneutral Cation-Chloride Cotransporters. Physiol. Rev. 85, 423-493 (2005).

52. S. Robinson, I. Mikolaenko, I. Thompson, M. L. Cohen, M. Goyal, Loss of Cation-Chloride Cotransporter Expression in Preterm Infants With White Matter Lesions: Implications for the Pathogenesis of Epilepsy. J. Neuropathol. Exp. Neurol. 69, 565-572 (2010).

53. K. T. Kahle, K. J. Staley, B. V. Nahed, G. Gamba, S. C. Hebert, R. P. Lifton, D. B. Mount, Roles of the cation-chloride cotransporters in neurological disease. Nat Clin Pr. Neuro. 4, 490-503 (2008).

54. S. Hamidi, M. Avoli, KCC2 function modulates in vitro ictogenesis. Neurobiol. Dis. 79, 5158 (2015).

55. G. B. Boylan, R. M. Pressler, Neonatal seizures: the journey so far. Semin. Neonatal Med. 18, 173-174 (2013).

56. G. B. Boylan, N. J. Stevenson, S. Vanhatalo, Monitoring neonatal seizures. Semin. Fetal. Neonatal Med. 18, 202-208 (2013).

57. G. B. Boylan, J. M. Rennie, R. M. Pressler, G. Wilson, M. Morton, C. D. Binnie, Phenobarbitone, neonatal seizures, and video-EEG. Arch. Dis. Child. - Fetal Neonatal Ed. 86, F165-F170 (2002).

58. M. Puskarjov, F. Ahmad, S. Khirug, S. Sivakumaran, K. Kaila, P. Blaesse, BDNF is required for seizure-induced but not developmental up-regulation of $\mathrm{KCC} 2$ in the neonatal hippocampus. Neuropharmacology. 88, 103-109 (2015).

59. S. N. Rakhade, F. E. Jensen, Epileptogenesis in the immature brain: emerging mechanisms. Nat. Rev. Neurol. 5, 380-391 (2009).

60. R. A. Cardarelli, K. Jones, L. I. Pisella, H. J. Wobst, L. J. McWilliams, P. M. Sharpe, M. P. Burnham, D. J. Baker, I. Chudotvorova, J. Guyot, L. Silayeva, D. H. Morrow, N. Dekker, S. Zicha, P. A. Davies, J. Holenz, M. E. Duggan, J. Dunlop, R. J. Mather, Q. Wang, I. Medina, N. J. Brandon, T. Z. Deeb, S. J. Moss, The small molecule CLP257 does not modify activity of the $\mathrm{K}+\mathrm{Cl}$ - co-transporter $\mathrm{KCC} 2$ but does potentiate GABAA receptor activity. Nat. Med. 23, 1394-1396 (2017).

61. M. Gagnon, M. J. Bergeron, J. Perez-Sanchez, I. Plasencia-Fernández, L.-E. Lorenzo, A. G. Godin, A. Castonguay, R. P. Bonin, Y. De Koninck, Reply to The small molecule CLP257 
does not modify activity of the $\mathrm{K}+-\mathrm{Cl}-$ co-transporter $\mathrm{KCC} 2$ but does potentiate GABA A receptor activity. Nat. Med. 23, 1396-1398 (2017).

752

753

754
62. B. Chen, Y. Li, B. Yu, Z. Zhang, B. Brommer, P. R. Williams, Y. Liu, S. V. Hegarty, S. Zhou, J. Zhu, H. Guo, Y. Lu, Y. Zhang, X. Gu, Z. He, Reactivation of Dormant Relay Pathways in Injured Spinal Cord by KCC2 Manipulations. Cell. 174, 521-535.e13 (2018).

63. J. C. S. Mapplebeck, L.-E. Lorenzo, K. Y. Lee, C. Gauthier, M. M. Muley, Y. De Koninck, S. A. Prescott, M. W. Salter, Chloride Dysregulation through Downregulation of KCC2 Mediates Neuropathic Pain in Both Sexes. Cell Rep. 28, 590-596.e4 (2019).

64. H. Saitsu, M. Watanabe, T. Akita, C. Ohba, K. Sugai, W. P. Ong, H. Shiraishi, S. Yuasa, H. Matsumoto, K. T. Beng, S. Saitoh, S. Miyatake, M. Nakashima, N. Miyake, M. Kato, A. Fukuda, N. Matsumoto, Impaired neuronal KCC2 function by biallelic SLC12A5 mutations in migrating focal seizures and severe developmental delay. Sci. Rep. 6, 30072 (2016).

65. V. Magloire, J. Cornford, A. Lieb, D. M. Kullmann, I. Pavlov, KCC2 overexpression prevents the paradoxical seizure-promoting action of somatic inhibition. Nat. Commun. 10, 1225 (2019).

66. L. A. Slaughter, A. D. Patel, J. L. Slaughter, Pharmacological Treatment of Neonatal Seizures: A Systematic Review. J. Child Neurol. 28, 351-364 (2013).

67. G. J. Markowitz, S. D. Kadam, D. R. Smith, M. V. Johnston, A. M. Comi, Different effects of high- and low-dose phenobarbital on post-stroke seizure suppression and recovery in immature CD1 mice. Epilepsy Res. 94, 138-148 (2011).

68. J. R. Farwell, Y. J. Lee, D. G. Hirtz, S. I. Sulzbacher, J. H. Ellenberg, K. B. Nelson, Phenobarbital for Febrile Seizures - Effects on Intelligence and on Seizure Recurrence. $N$. Engl. J. Med. 322, 364-369 (1990).

69. S. Sulzbacher, J. R. Farwell, N. Temkin, A. S. Lu, D. G. Hirtz, Late cognitive effects of early treatment with phenobarbital. Clin. Pediatr. (Phila.). 38, 387-394 (1999).

70. X. Tang, Neuronal Chloride Transporters in Health and Disease (Elsevier, ed. 1, 2020).

71. B. J. Sullivan, S. D. Kadam, in Experimental and Translational Models to Screen Drugs Effective Against Seizures and Epilepsy (Springer Nature, 2020), Neuromethods book series. 


\section{Figure 1}
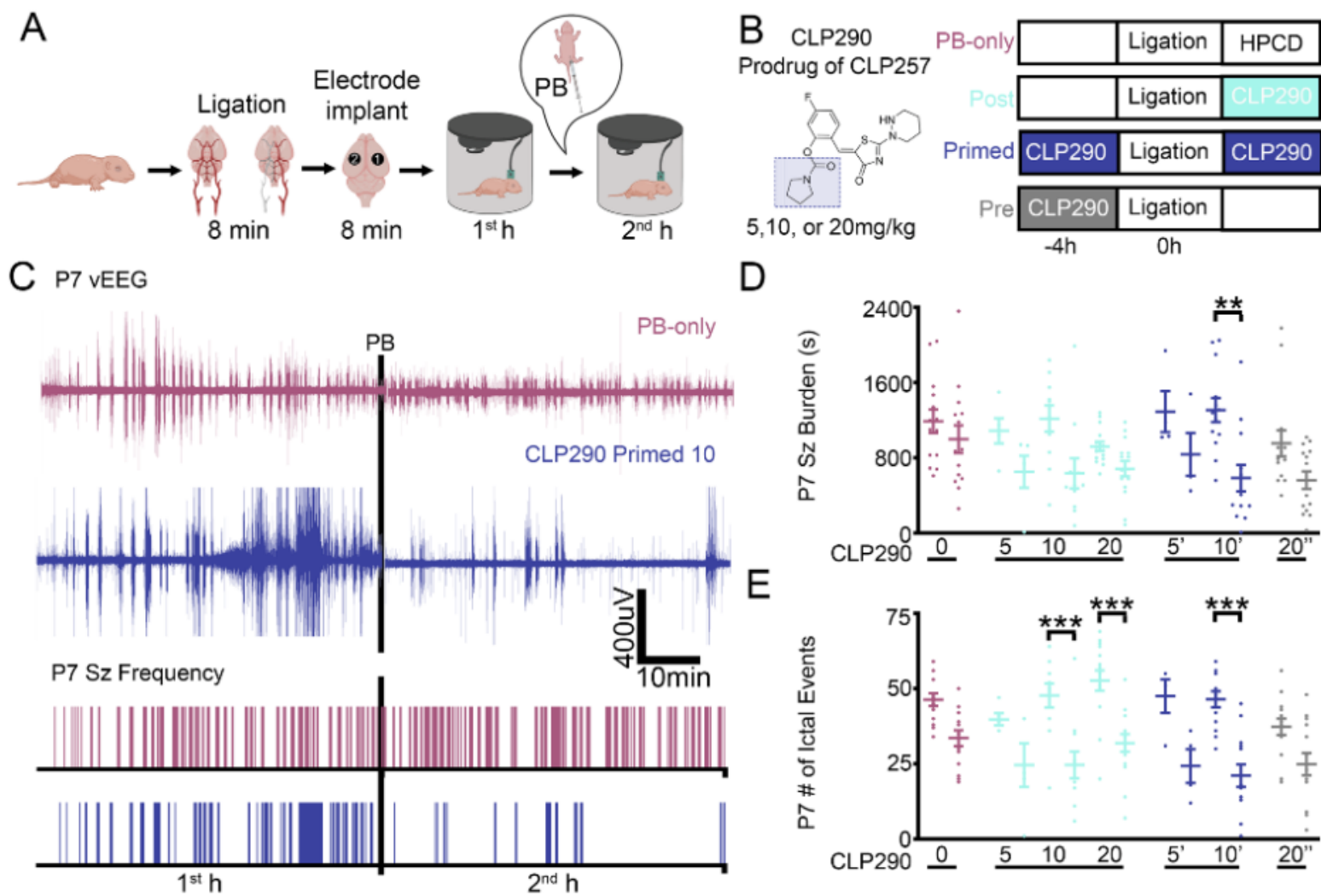

G

CLP257

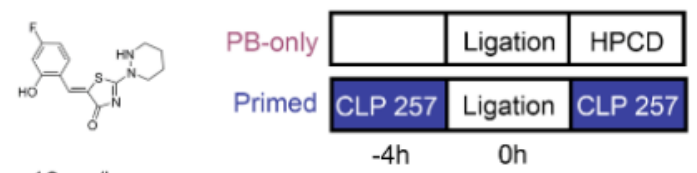

F

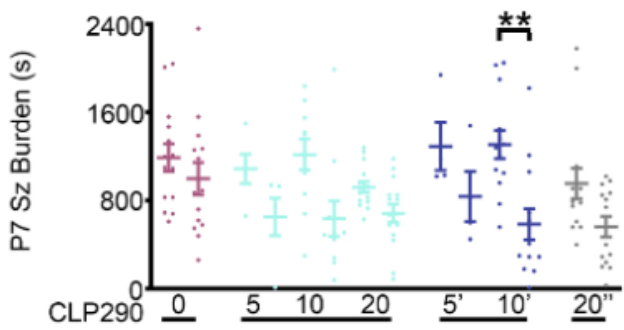

$E$



$10 \mathrm{mg} / \mathrm{kg}$

$\mathrm{H}$
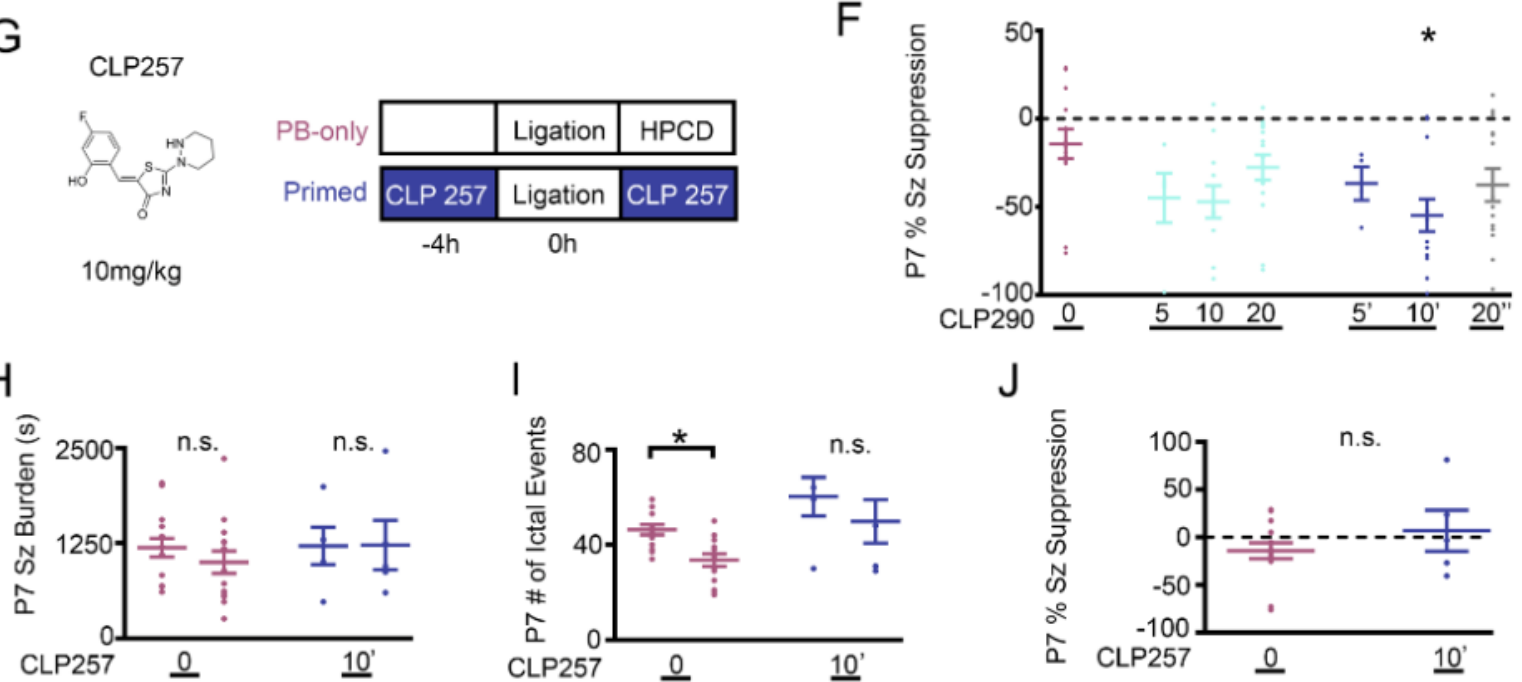

K

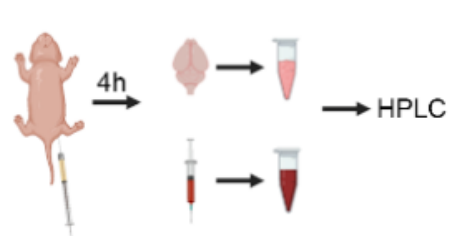

L

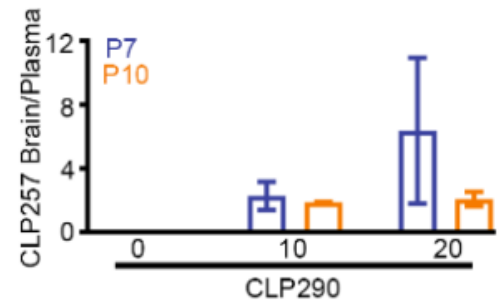

J

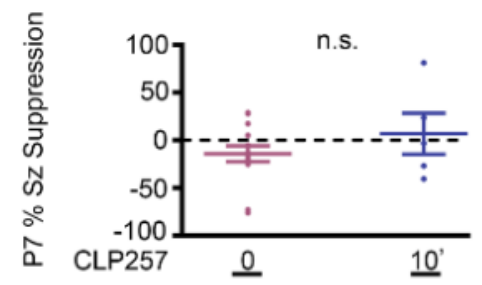

M

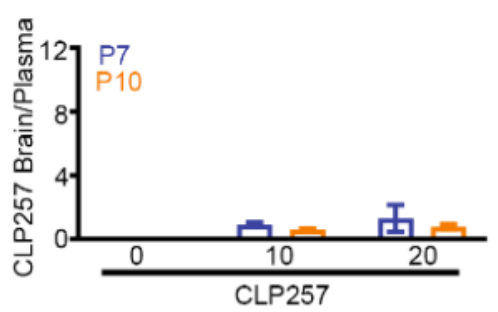


Fig. 1. CLP290 rescued phenobarbital-resistant neonatal seizures in P7-CD1 mice.

(A) Experimental design of a P7 CD-1 mouse model of ischemic neonatal seizures with continuous vEEG. Recording (1) and reference (2) electrodes over bilateral parietal cortices, with a ground electrode over the rostrum. (B) Doses and treatment protocols to evaluate CLP290, a prodrug of the proposed KCC2 functional enhancer CLP257. (C) Representative EEG traces and seizure frequency raster plots of a PB-only and CLP290

791 10' pup. Black bars represent a loading dose of PB (25mg/kg; intraperitoneal injection). (D) $1^{\text {st }}$ and $2^{\text {nd }}$ hour seizure burdens, (E) $1^{\text {st }}$ and $2^{\text {nd }}$ hour ictal events, and $(\mathbf{F}) 1^{\text {st }} v s .2^{\text {nd }}$ 793 hour percent seizure suppression after P7 unilateral carotid ligation. Percent seizure

794 suppression was analyzed by one-way ANOVA vs. PB-only. PB-only $n=14$; CLP290 5 $795 n=5$; CLP290 10 n=11; CLP290 20 n=15; CLP290 5’n=4; CLP290 10’n=13; CLP290 20' $796 \mathrm{n}=14$. (G) Doses and treatment protocols to evaluate CLP257 ( $\mathrm{n}=5)$. (H) Seizure burdens, 797 (I) ictal events, and (J) percent seizure suppression at P7 after unilateral carotid ligation. 798 (K) Experimental paradigm to investigate the pharmacokinetic profile of CLP290 and 799 CLP257. (L) CLP257 brain to plasma ratio after CLP290 administration (I.P., $n=2$ per 800 group). (M) CLP257 brain to plasma ratio after CLP257 administration (I.P.; $n=2$ per 801 group). Plots show all data points with means \pm SEM. ${ }^{*} \mathrm{P}<0.05$; ${ }^{* *} \mathrm{P}<0.01$; ${ }^{* *} \mathrm{P}<0.001$, two802 way ANOVA. 


\section{Figure 2}

A



C

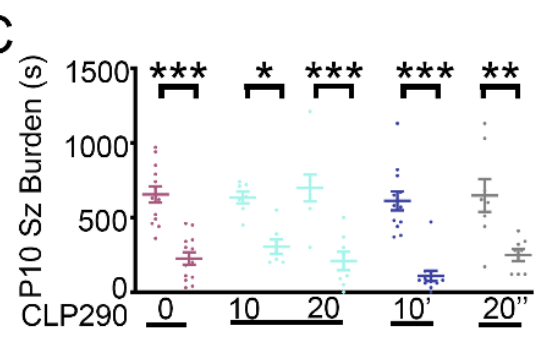

B
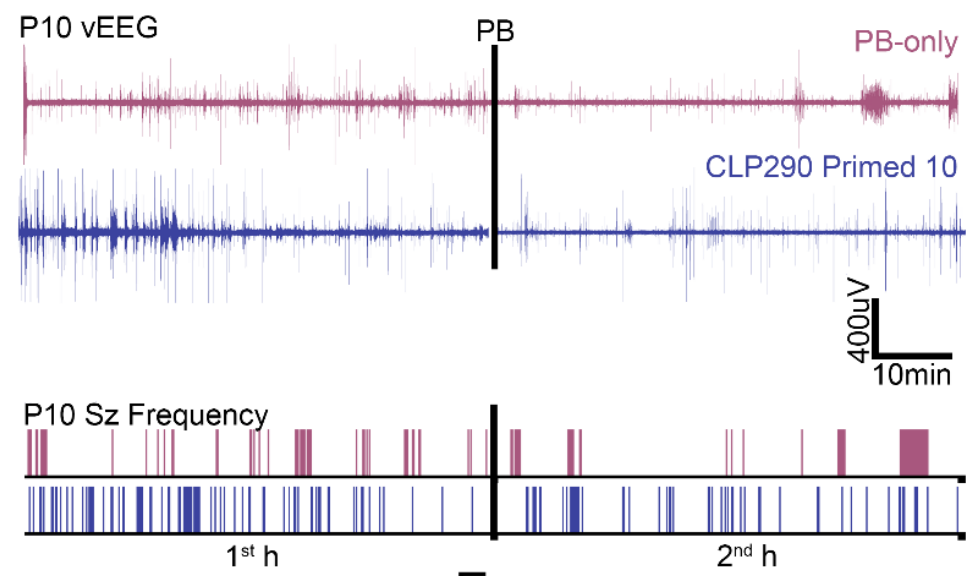

D

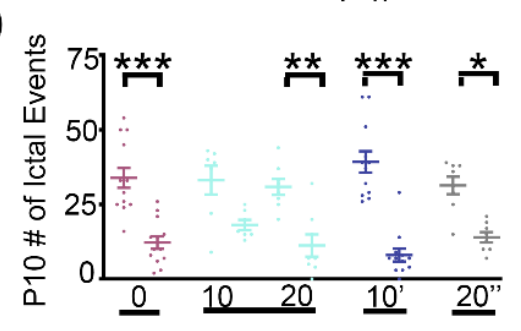

E 응

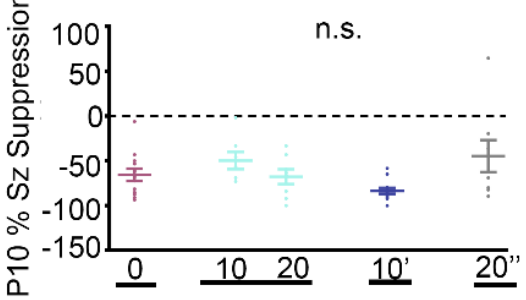


821 Fig.2. Ischemic neonatal seizures in P10_-CD1 pups were phenobarbital822 responsive. (A) Doses and treatment protocols to evaluate CLP290 in P10 CD-1 pups.

823 (B) Representative EEG traces and seizure frequency raster plots of a P10 phenobarbital824 only and CLP290 10' pup. Black bars represent a loading dose of PB (25mg/kg; 825 intraperitoneal injection). (C) $1^{\text {st }}$ and $2^{\text {nd }}$ hour seizure burdens, (D) $1^{\text {st }}$ and $2^{\text {nd }}$ hour ictal 826 events. (E) CLP290 does not improve seizure suppression at P10. $1^{\text {st }}$ vs. $2^{\text {nd }}$ hour percent 827 seizure suppression after unilateral carotid ligation at P10. Percent seizures suppression 828 was analyzed by one-way ANOVA vs. PB-only. Plots show all data points with means 829 ISEM. ${ }^{*} \mathrm{P}<0.05 ;{ }^{* *} \mathrm{P}<0.01 ;{ }^{* *} \mathrm{P}<0.001$, two-way ANOVA. PB-only; $\mathrm{n}=13$; CLP290 $10 \mathrm{n}=7$;

830 CLP290 20 n=8; CLP290 10’ n=12; CLP290 20” n=8. 


\section{Figure 3}

A

P7

$\mathrm{F}$

P10
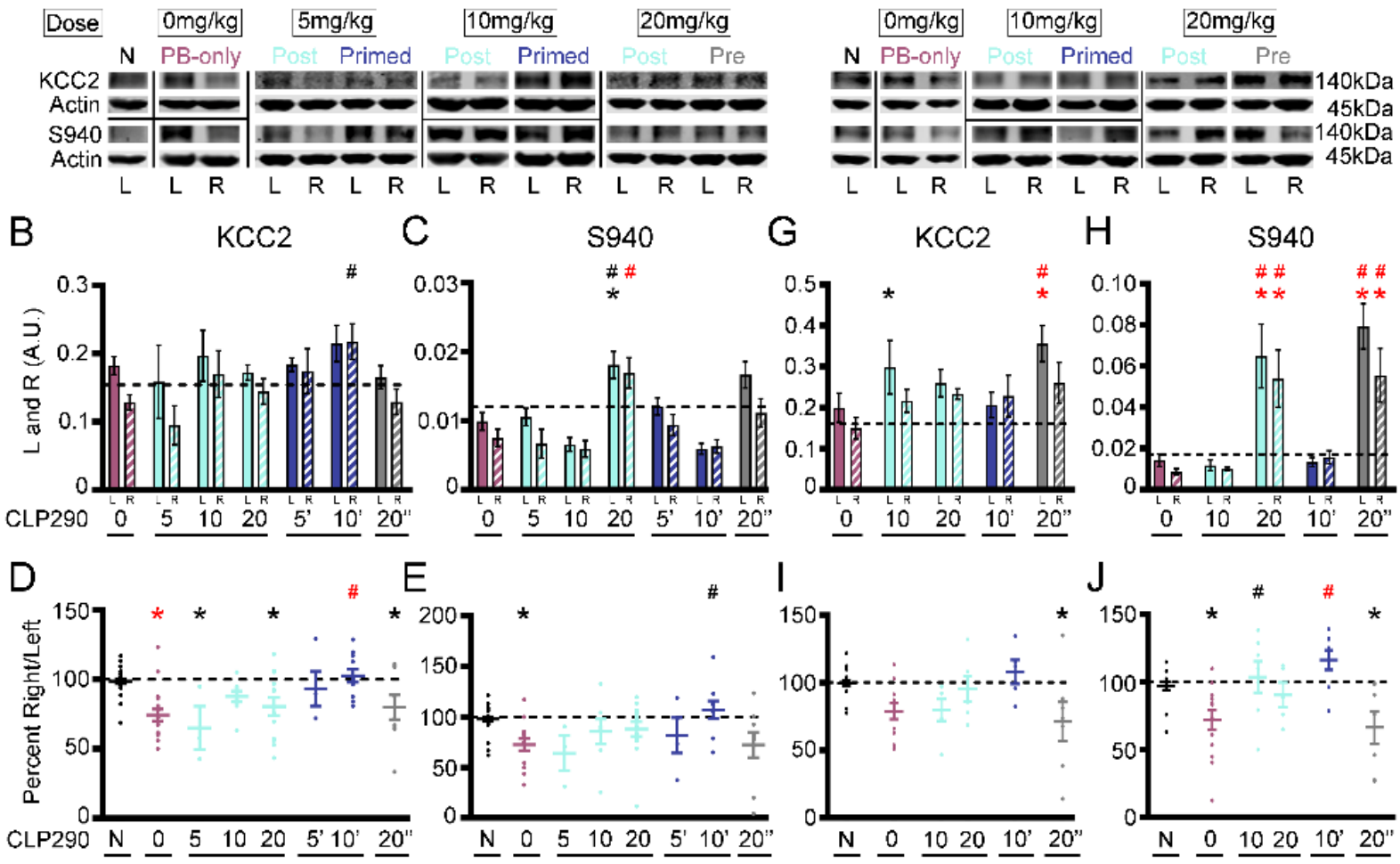

K

P7

$\mathrm{P}$
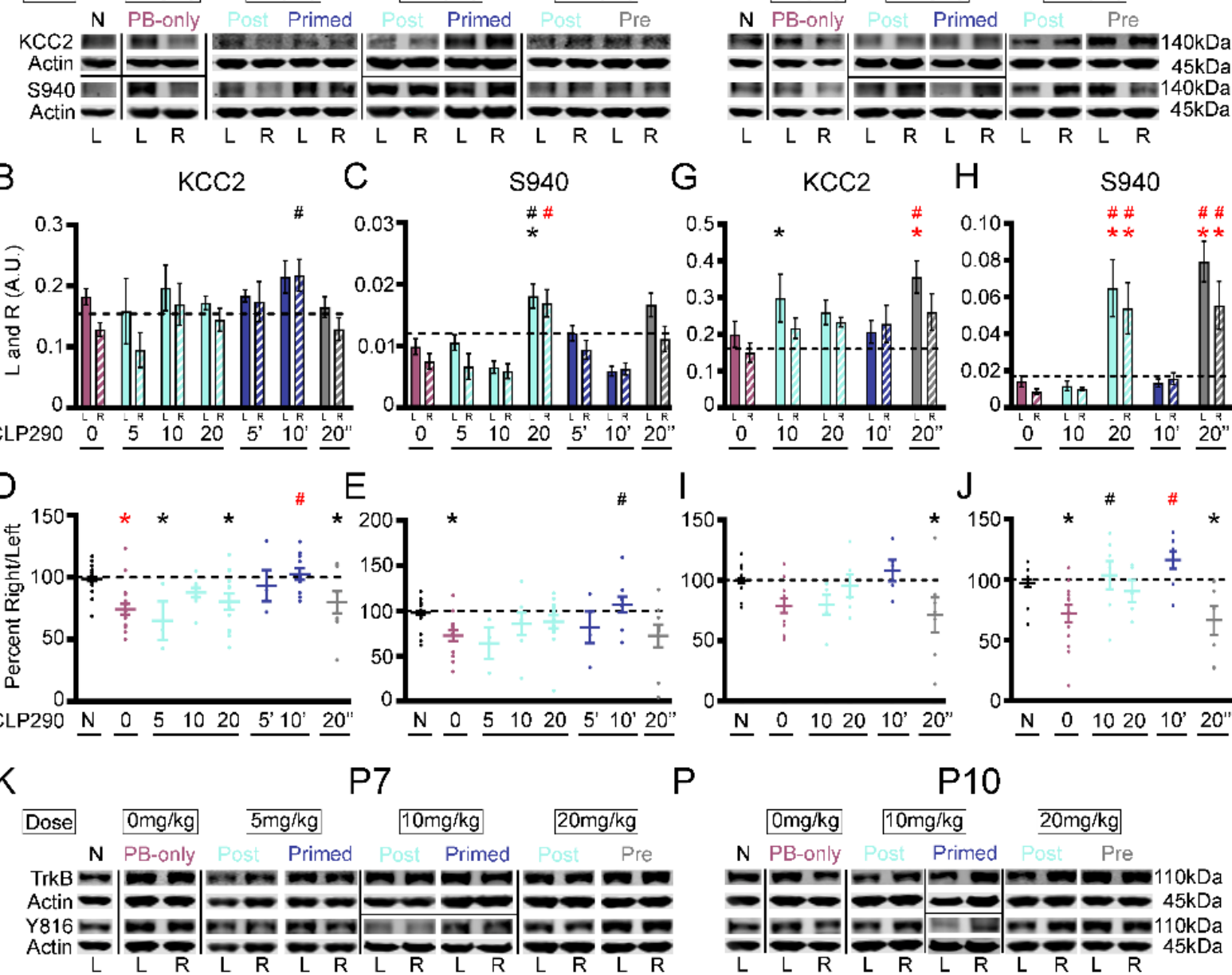

$\mathrm{L}$

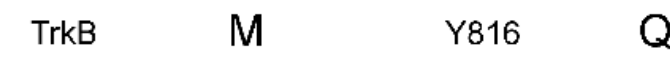

Q TrkB
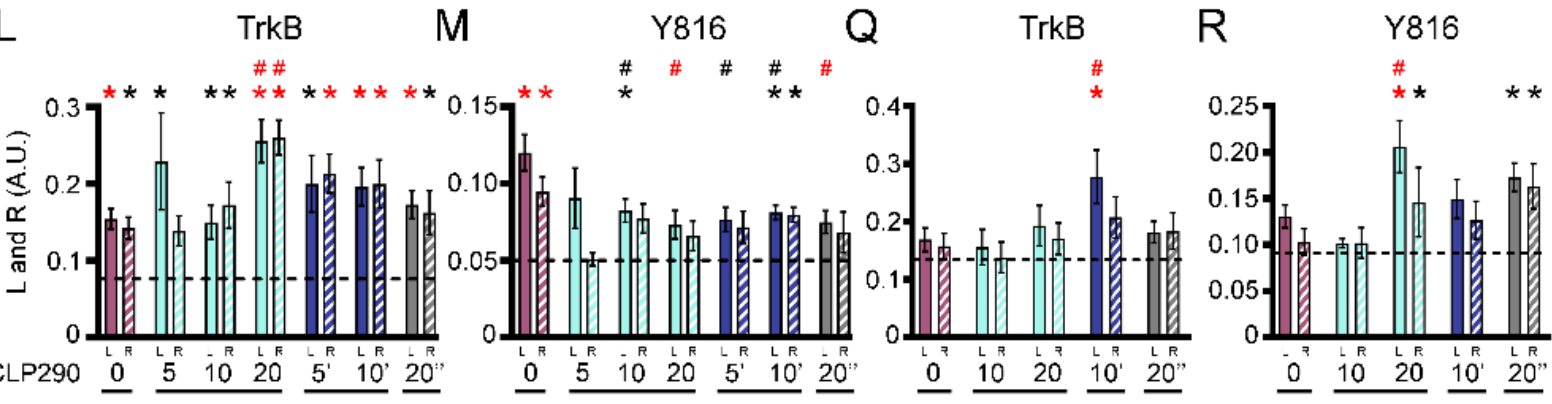

$\mathrm{N}$

$\mathrm{O}$

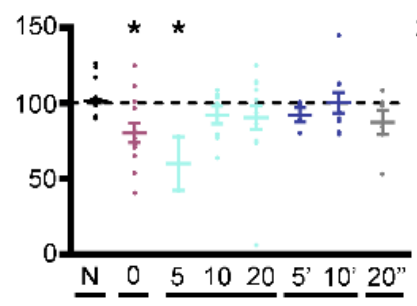

$S$

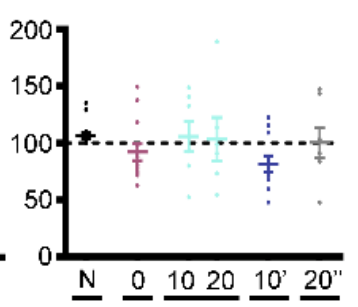

$T$

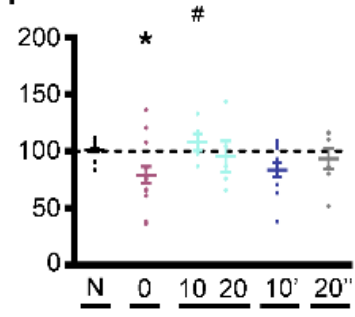


847 Fig. 3. CLP290 rescued post-ischemic KCC2 downregulation but not TrkB

848 activation. (A) Representative Western blots of KCC2 and S940 protein expression 24h

849 after P7 ischemic seizures. (B) KCC2 and (C) S940 expression in left (L) and right (R)

850 hemispheres. (D) KCC2 and (E) $\mathrm{S940}$ expression as percent ipsilateral/contralateral

851 (R/L). (F) Representative Western blots of KCC2 and S940 expression 24h after P10

852 ischemic seizures. (G) KCC2 and (H) S940 expression in L and R hemispheres. (I) KCC2

853 and (J) S940 expression as percent R/L. (K) Representative Western blots of TrkB and

854 Y816 expression 24h after P7 ischemic seizures. (L) TrkB and (M) Y816 expression in L

855 and $\mathrm{R}$ hemispheres. (N) TrkB and (O) Y816 expression as percent R/L. (P)

856 Representative Western blots of TrkB and Y816 expression 24h after P10 ischemic

857 seizures. (Q) TrkB and (R) Y816 expression in $L$ and R hemispheres. (S) TrkB and (T)

858 Y816 expression as percent R/L. Data plots show means \pm SEM. All proteins of interest

859 were normalized to housekeeping protein $\beta$-actin. Phosphoproteins were normalized to

860 their respective total protein. ${ }^{*} \mathrm{P}<0.05$ and ${ }^{*} \mathrm{P}<0.001$ by 1 -way ANOVA vs. Naive. \#P $<0.05$

861 and $\# P<0.001$ vs. PB-Only. P7 pups: Naïve $n=27$; PB-only $n=18 ; 5$ Post $n=3 ; 10$ Post

862 n=9; 20 Post n=13; 5’, n=4; 10’, n=11; 20 Pre n=9. P10 pups: Naïve $n=18$, PB-only $n=11$,

86310 Post $n=6,20$ Post $n=6,10$ Primed $n=5,20$ Pre $n=7$.

864

865

866

867

868 
A

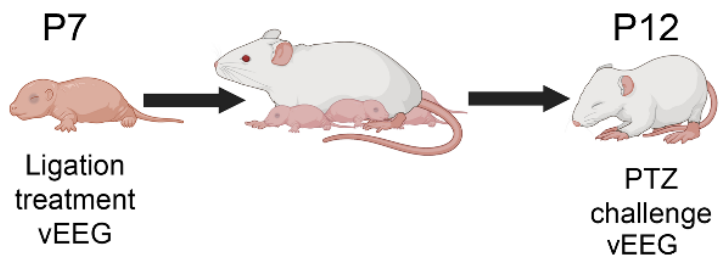

B

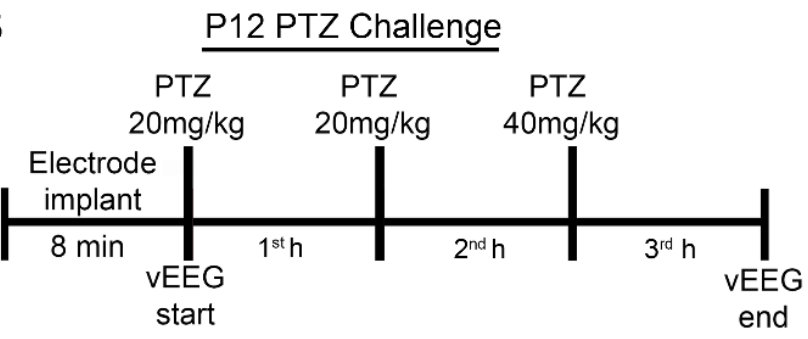

C
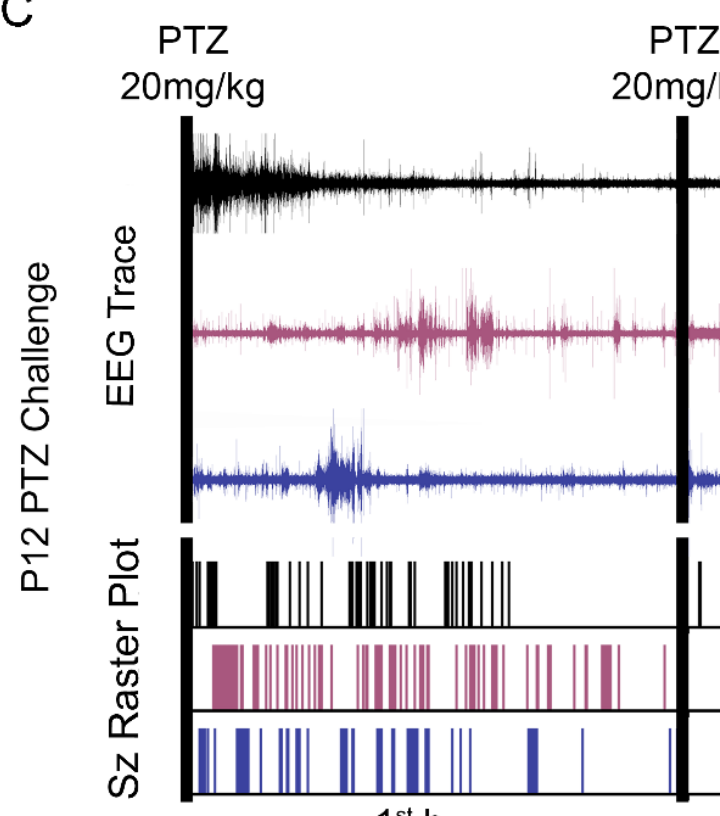

$$
1^{\text {st } h}
$$

PTZ $20 \mathrm{mg} / \mathrm{kg}$

D P12 Sz Burden (s)

E

$2^{\text {nd }} \mathrm{h}$

P12 Sz Duration (s)
PTZ $40 \mathrm{mg} / \mathrm{kg}$

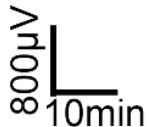

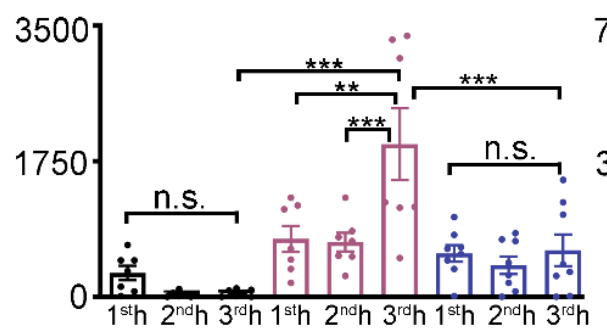
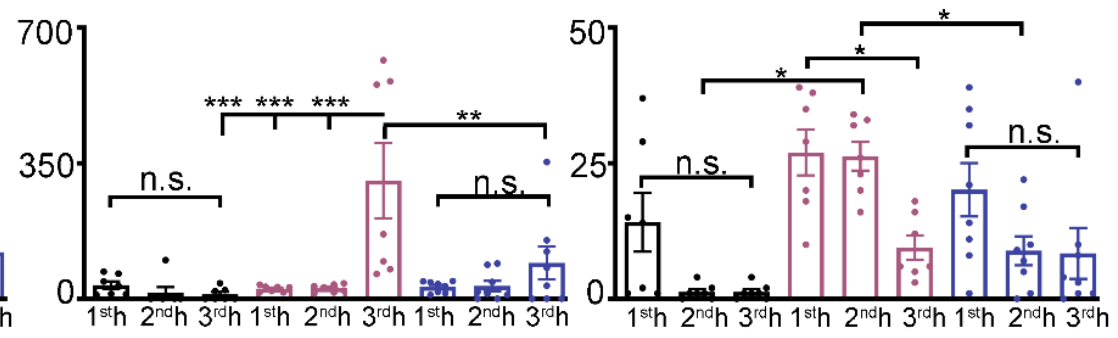
875 Fig. 4. CLP290-mediated regression of epileptogenesis detected using a PTZ

876 challenge. (A) Schematic to investigate the developmental benefits of CLP290 10'

877 treatment for P7 ischemic seizures. (B) P12 pentylenetetrazol (PTZ) challenge to

878 evaluate epileptogenesis. (C) Representative EEG traces and seizure frequency raster

879 plots for P12 pups that underwent Naïve, PB-only, or CLP290 10' treatment at P7. Black

880 bars indicate intraperitoneal PTZ injections. (D) $1^{\text {st }}, 2^{\text {nd }}$, and $3^{\text {rd }}$ hour seizure burdens at

881 P12 in CD-1 mice after PTZ injections. (E) Total electrographic seizure burdens over the

882 three hours of vEEG recording. (F) Total seizure events over three hours of vEEG

883 recording. Data plots show all data points with means \pm SEM. ${ }^{*} \mathrm{P}<0.05 ;{ }^{* *} \mathrm{P}<0.01$;

$88{ }^{* * *} \mathrm{P}<0.001$ by 2-way ANOVA. Naïve $n=7$; PB-only $n=7 ;$ CLP290 10’ $n=8$.

885

886

887

888

889

890

891

892

893

894

895

896

897 


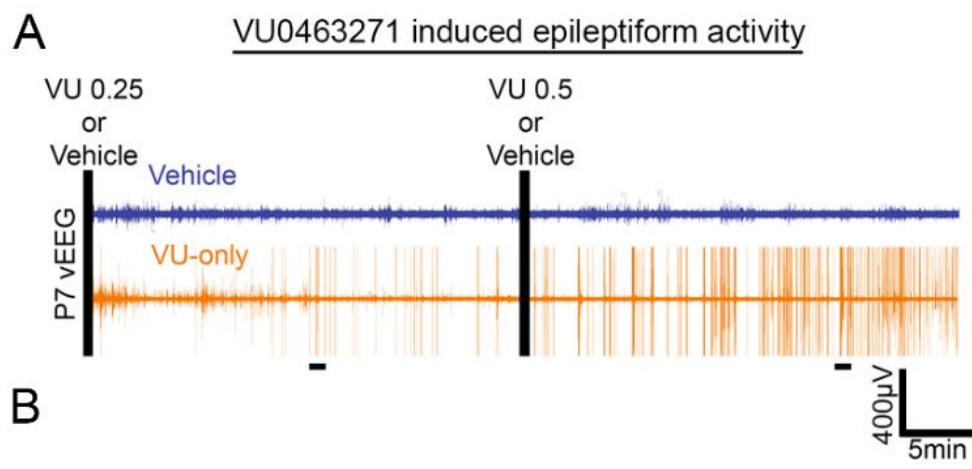

C
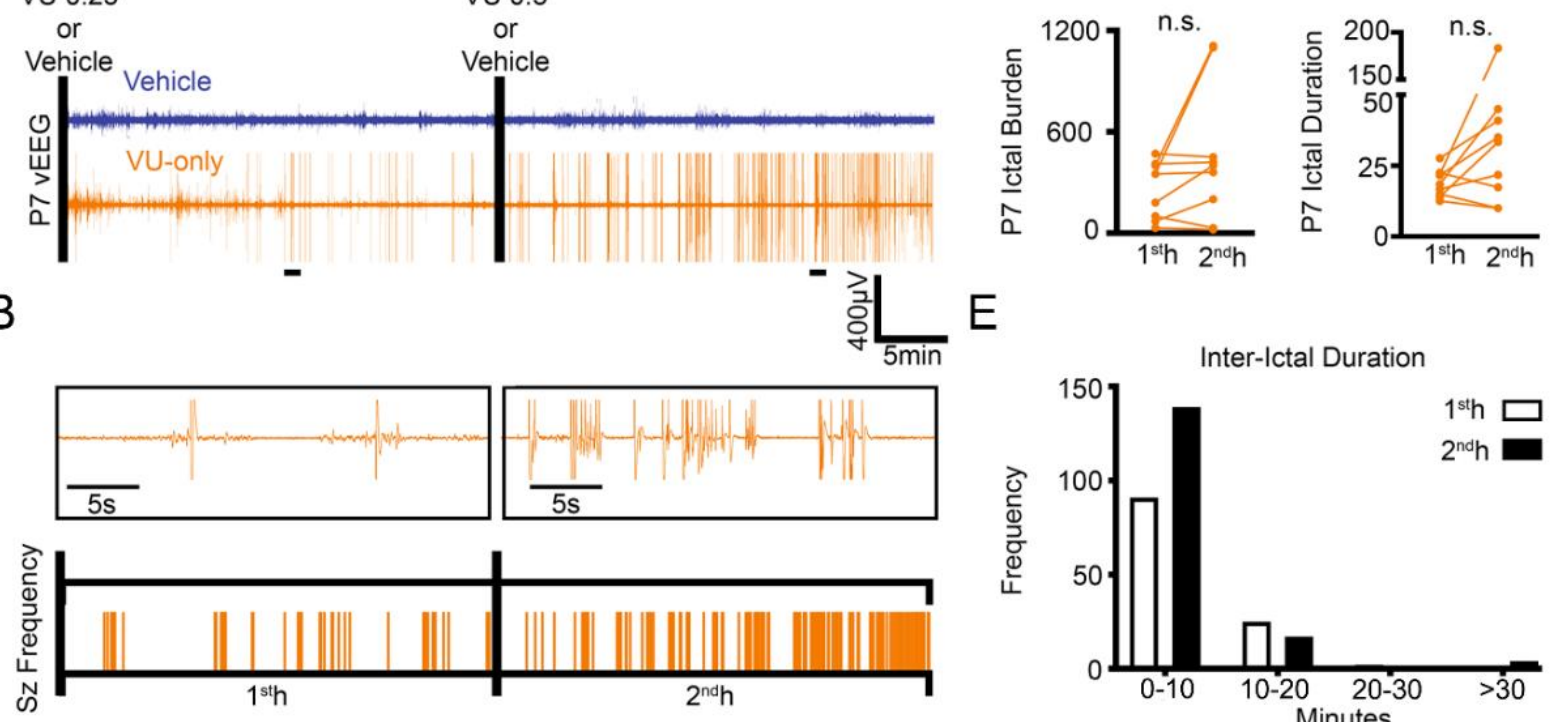

$E$



$\mathrm{F}$

G
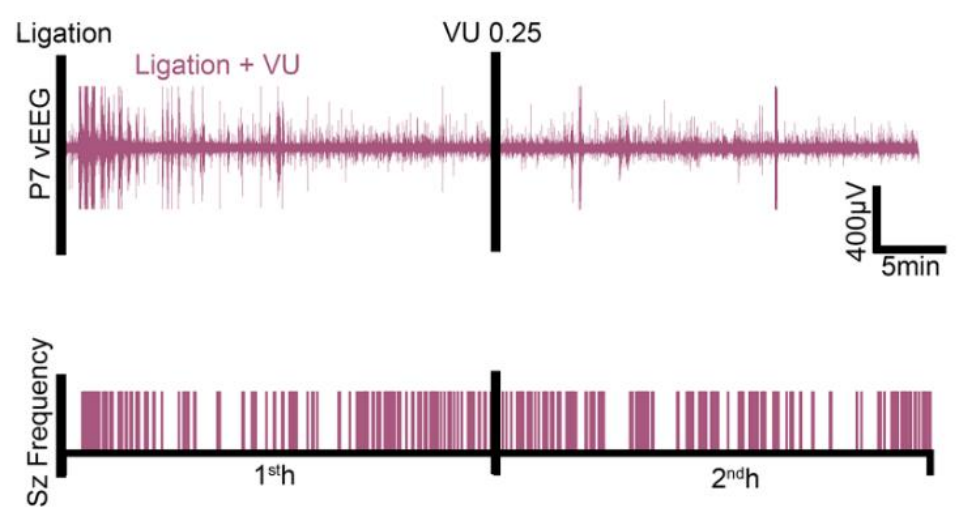

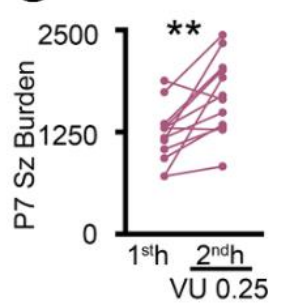

I Sz Burden

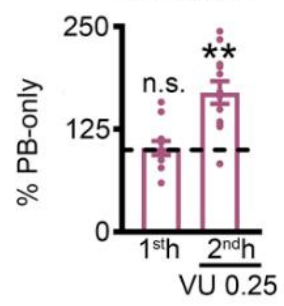

$\mathrm{H}$

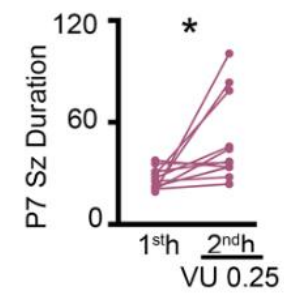

$J$ Sz Duration

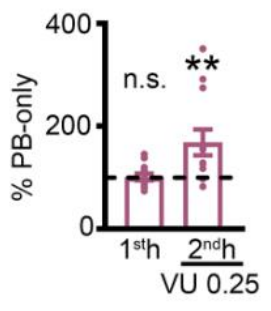


906 Fig. 5. Selective KCC2 antagonist VU induced spontaneous epileptiform

907 discharges in P7 pups. (A) Representative EEG traces and (B) ictal event frequency

908 raster plots from P7 CD-1 pups that either underwent vehicle or VU0463271 (VU)

909 administration. Black bars represent intraperitoneal injections. Expanded timescales

910 show VU induced epileptiform activity in the first and second hour. (C) $1^{\text {st }}$ vs. $2^{\text {nd }}$ hour ictal

911 burden and (D) ictal duration after VU administration. (E) Total frequency distribution for

912 all interictal durations in P7 CD-1 pups administered VU. Vehicle $(n=4)$ VU administration

913 (n=8). (F-J) VU aggravated ischemic neonatal seizure burdens. (F) Representative EEG

914 trace and seizure frequency raster plot of a P7 CD-1 pup that underwent unilateral carotid

915 ligation with administration of $\mathrm{VU} 0.25 \mathrm{mg} / \mathrm{kg}$ at $1 \mathrm{~h}$ (denoted by black bar). (G) $1^{\text {st }}$ and $2^{\text {nd }}$

$916 \mathrm{~h}$ seizure burden and $(\mathbf{H}) 1^{\text {st }}$ and $2^{\text {nd }} \mathrm{h}$ seizure duration for $\mathrm{P} 7 \mathrm{CD}-1$ pups that underwent

917 unilateral carotid ligation with administration of VU0.25mg/kg at $1 \mathrm{~h}$. (I) $1^{\text {st }}$ and $2^{\text {nd }}$ hour

918 seizure burden and $(\mathbf{J})$ duration plotted as percent PB-only. Data plots show all data

919 points with means \pm SEM. ${ }^{* *} \mathrm{P}<0.05$ and ${ }^{* *} \mathrm{P}<0.01$ by two-tailed paired t-test. Ligation +

920 VU $n=12$.

921

922

923

924

925

926

927

928 


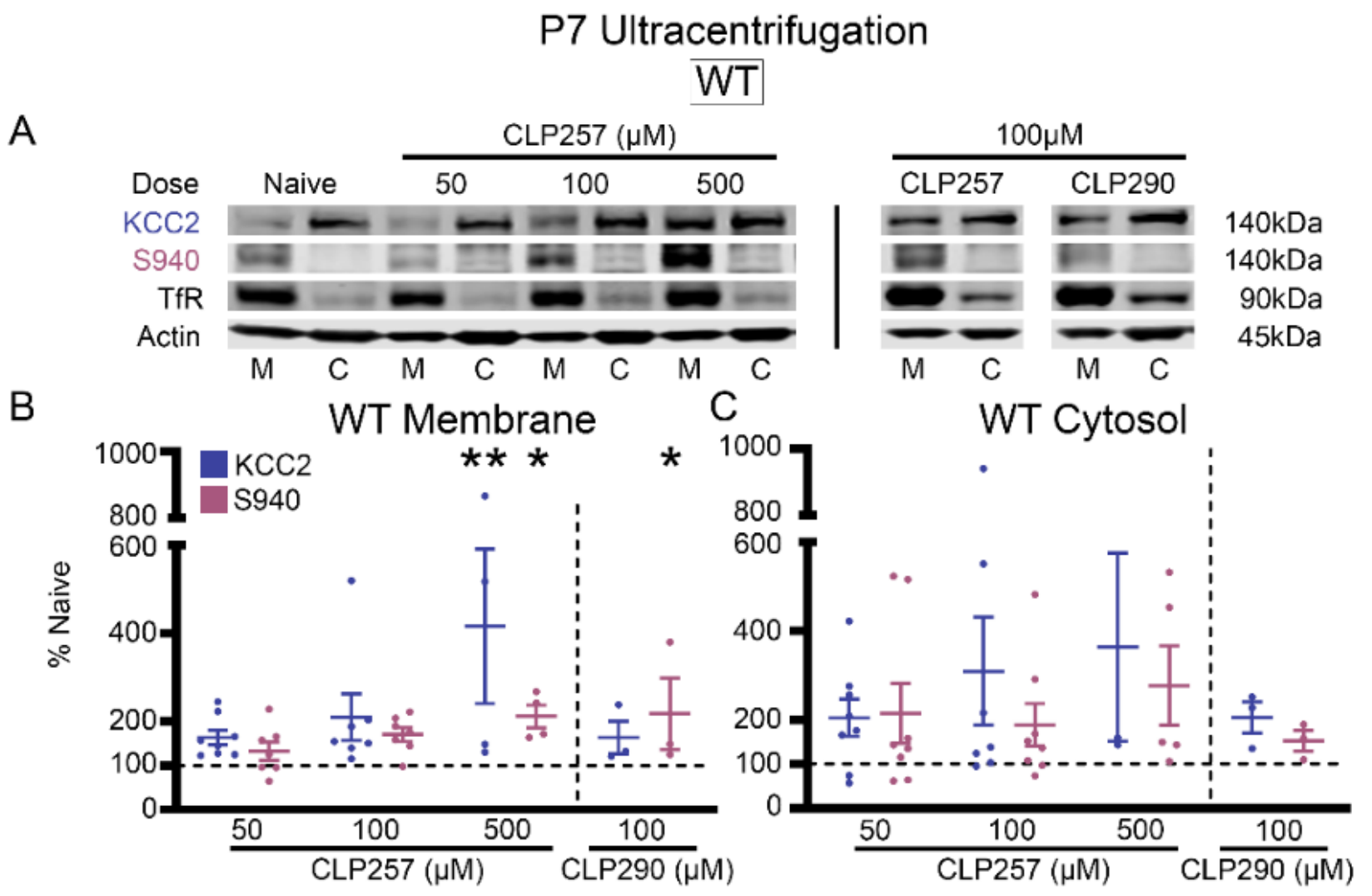

S940A

D WT S940A Knock-in E

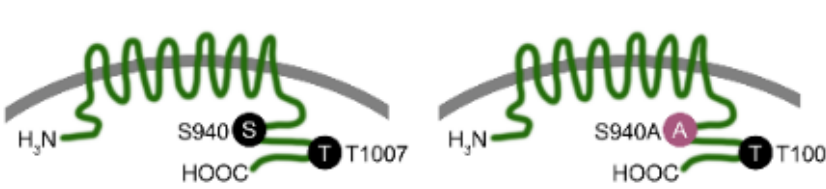

$\mathrm{F}$

930

Dose Naive $\quad 50 \quad 100 \quad 500$

$\mathrm{KCC} 2 \longrightarrow-\cdots=-$
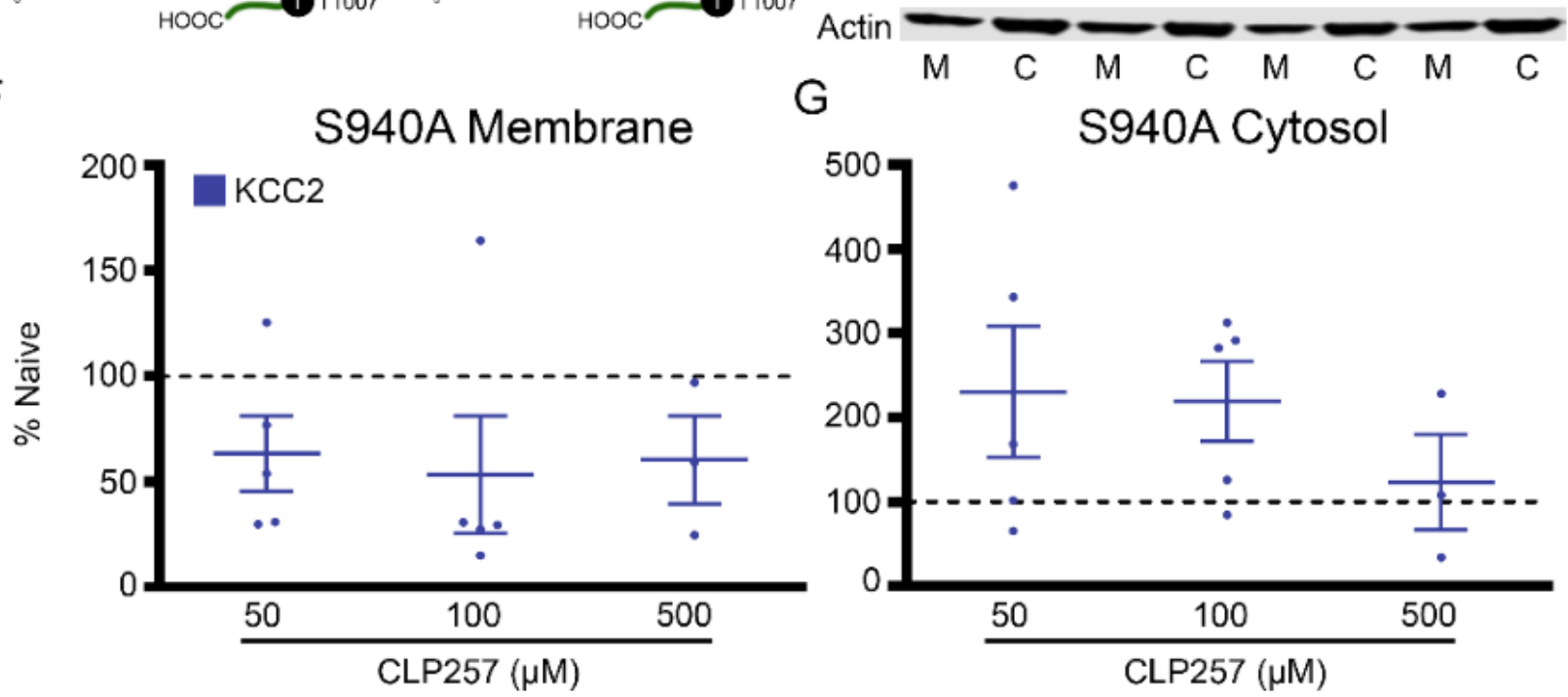
931 Fig. 6. CLP257 upregulated membrane KCC2 expression and 5940

932 phosphorylation. (A) KCC2 and S940 protein expression in the plasma membrane (M)

933 and cytosol (C) for all treated P7 wildtype (WT) brain slices. (B) KCC2 and S940 protein

934 expression in the membrane and $(\mathbf{C})$ cytosol for all treatment groups plotted as percent

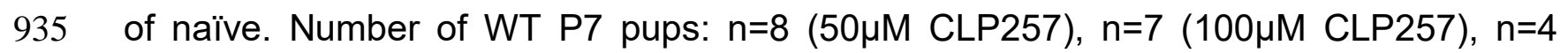

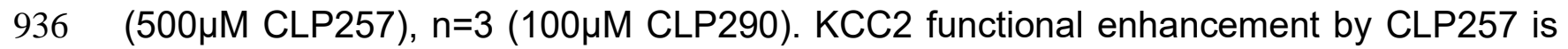

937 dependent upon the phosphorylation of S940. (D) Graphical representation of S940A ${ }^{+/+}$

938 knock-in mutant mice (36). (E) KCC2 expression in the plasma membrane and cytosol

939 for all treated brain slices from S940A+/+ P7 pups. (F) KCC2 expression in the membrane

940 and $(\mathbf{G})$ cytosol for all $S 940 \mathrm{~A}^{+/+}$treatment groups plotted as percent of naïve. All proteins

941 of interest in the cytosol were normalized to housekeeping protein $\beta$-actin. All proteins of

942 interest in the plasma membrane were normalized to transferrin (TfR). Phosphoproteins

943 were normalized to their respective total protein. Data plots show all data points with

944 means \pm SEM. * $\mathrm{P}<0.05$ and ${ }^{* *} \mathrm{P}<0.01$ by 1 -way ANOVA. S940A ${ }^{+/+} \mathrm{P} 7$ pups: $\mathrm{n}=5(50 \mu \mathrm{M}$

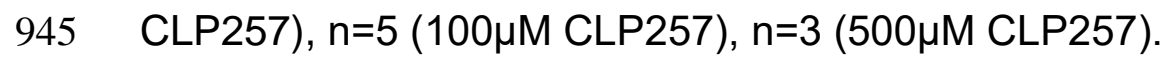




\section{$954 \quad$ Figure 7}

A

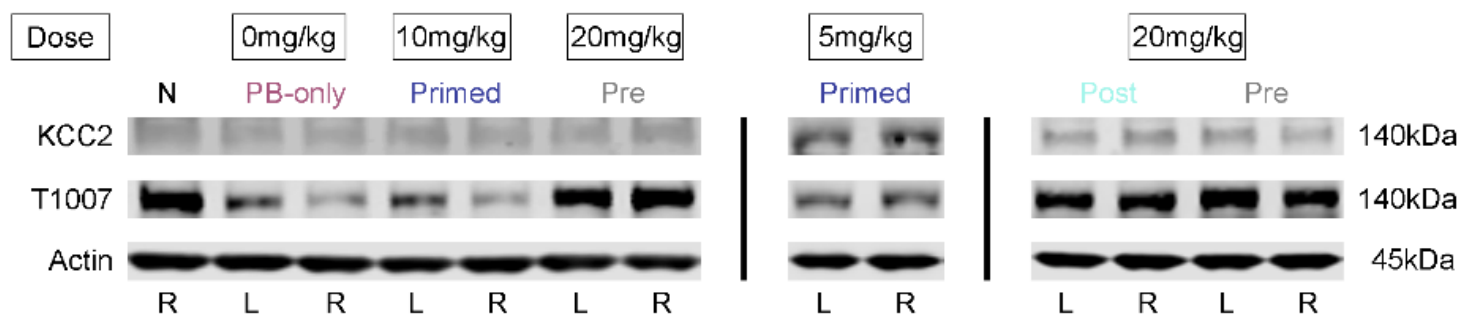

B

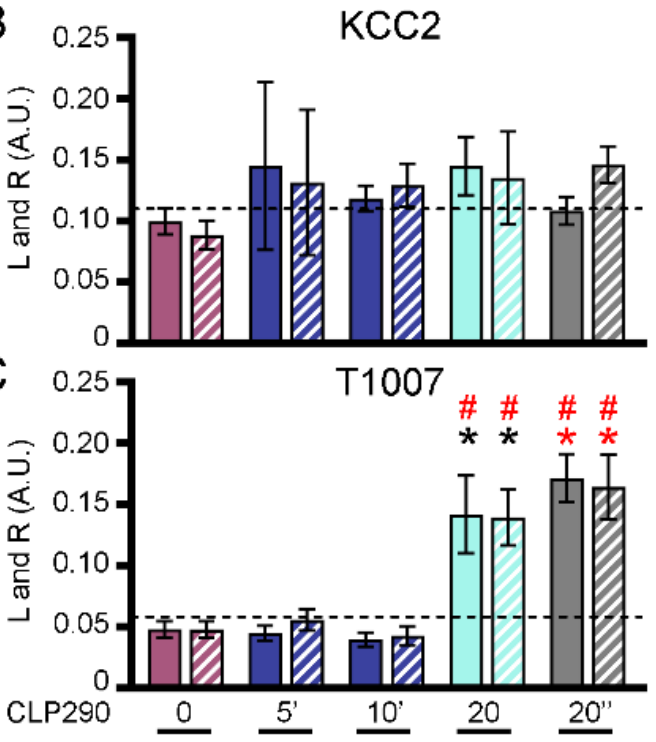

D

T1007/KCC2

E
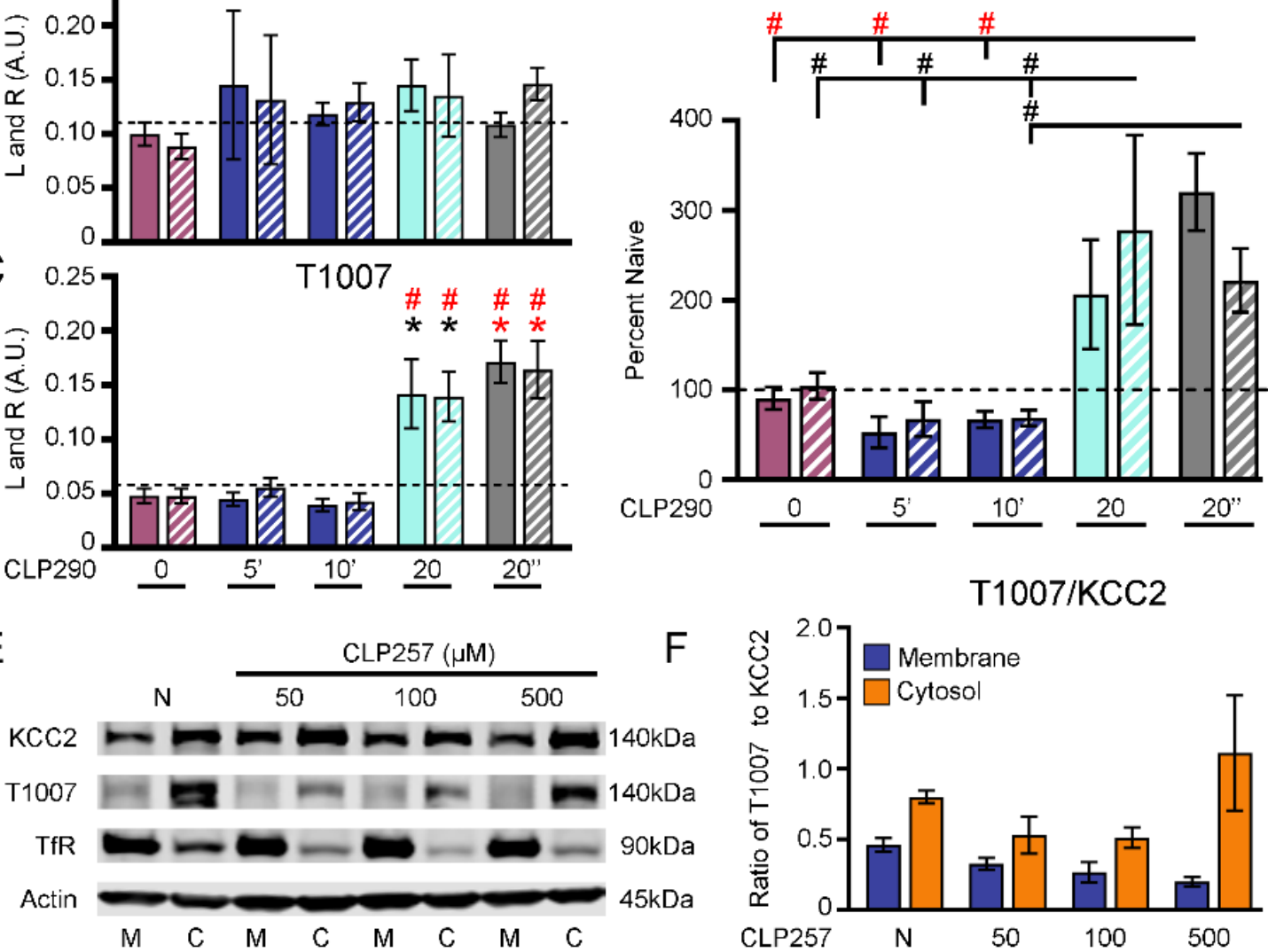

\section{T1007/KCC2}

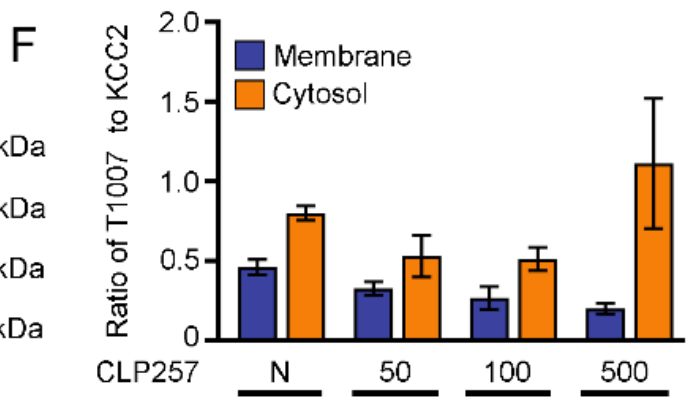

G

Membrane

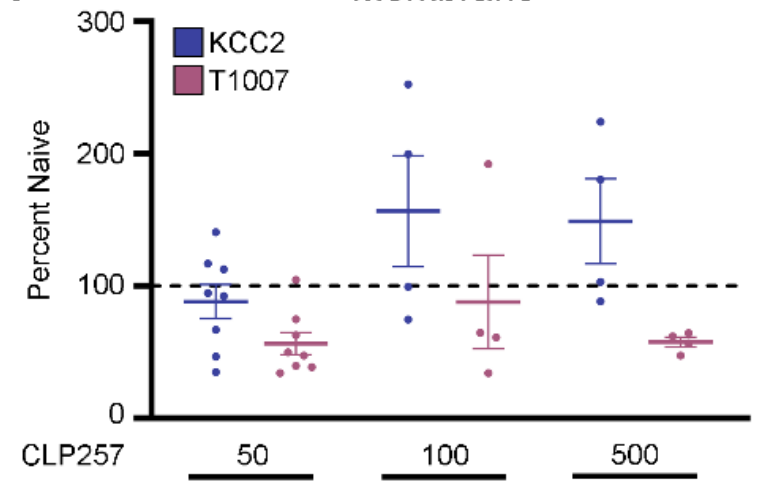

$\mathrm{H}$

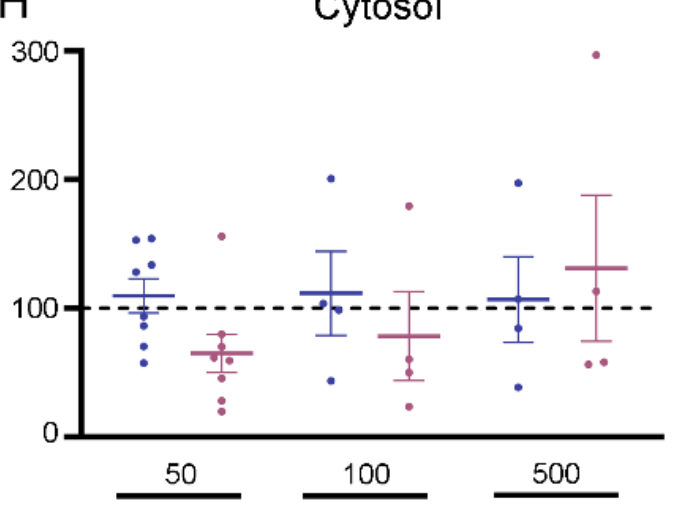


956 Fig. 7. Bolus administration of CLP290 $20 \mathrm{mg} / \mathrm{kg}$ induced homeostatic upregulation

957 of T1007 phosphorylation. (A) Representative western blot of KCC2 and T1007

958 expression 24h after ischemic neonatal seizures. (B) KCC2 and (C) T1007 expression in

959 left (L) and right (R) hemispheres. (D) T1007/KCC2 ratios plotted as percent naïve for $\mathrm{L}$

960 and R hemispheres. Naïve $n=10$ PB-only; $n=14$; CLP290 5' $n=3$; CLP290 10' n=9;

961 CLP290 20 n=4; CLP290 20" n=8. (E) Representative western blot of KCC2 and T1007

962 expression at the membrane (M) and cytosol (C) in CLP257 treated slices. (F) T1007-

963 KCC2 ratio for M and C for CLP257 treated slices. (G) Membrane KCC2 and T1007

964 plotted as percent naïve. (H) Cytosol KCC2 and T1007 plotted as percent naïve. ${ }^{*} \mathrm{P}<0.05$

965 and ${ }^{*} \mathrm{P}<0.001$ by one-way ANOVA vs Naive. $\# \mathrm{P}<0.05$ and $\# \mathrm{P}<0.001$ by one-way ANOVA

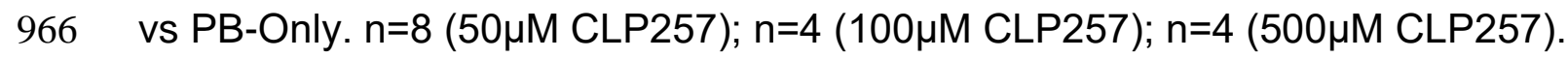

967

968

969

970

971

972

973

974

975

976

977

978 
A

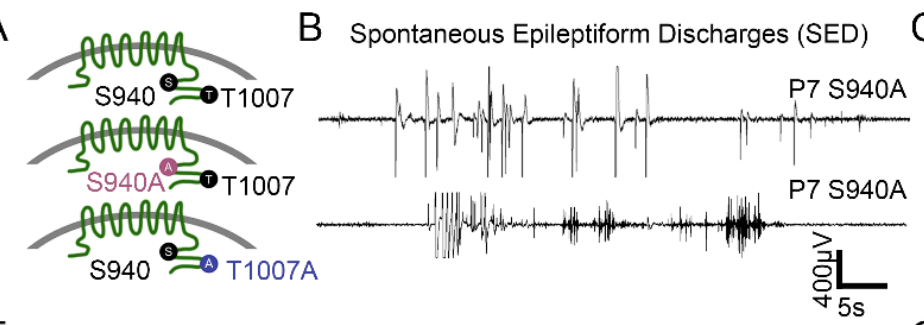

E Ligat

E Lig

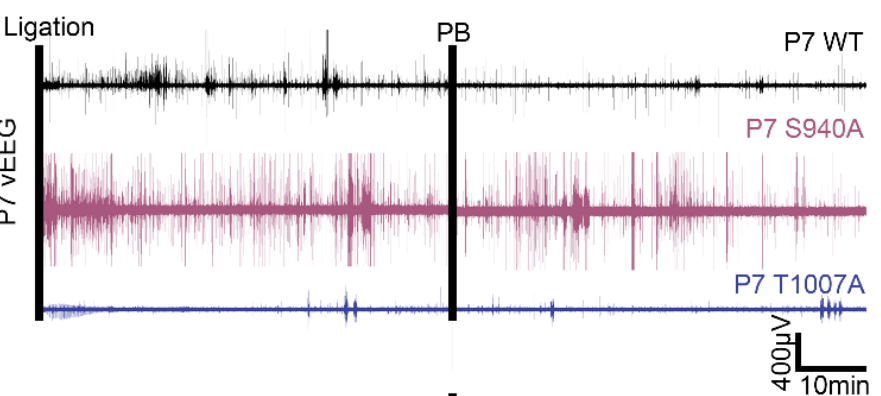

$\mathrm{F}$

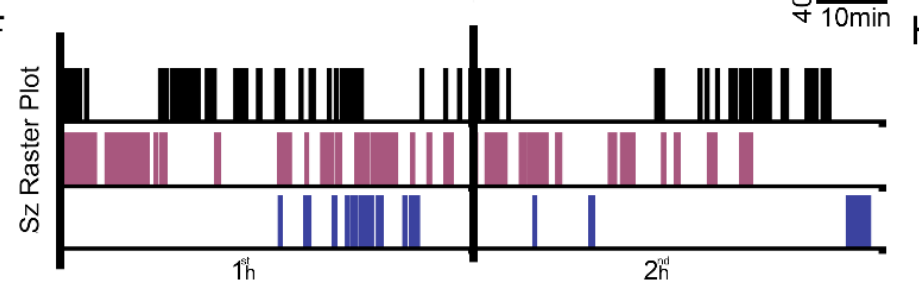

$J$
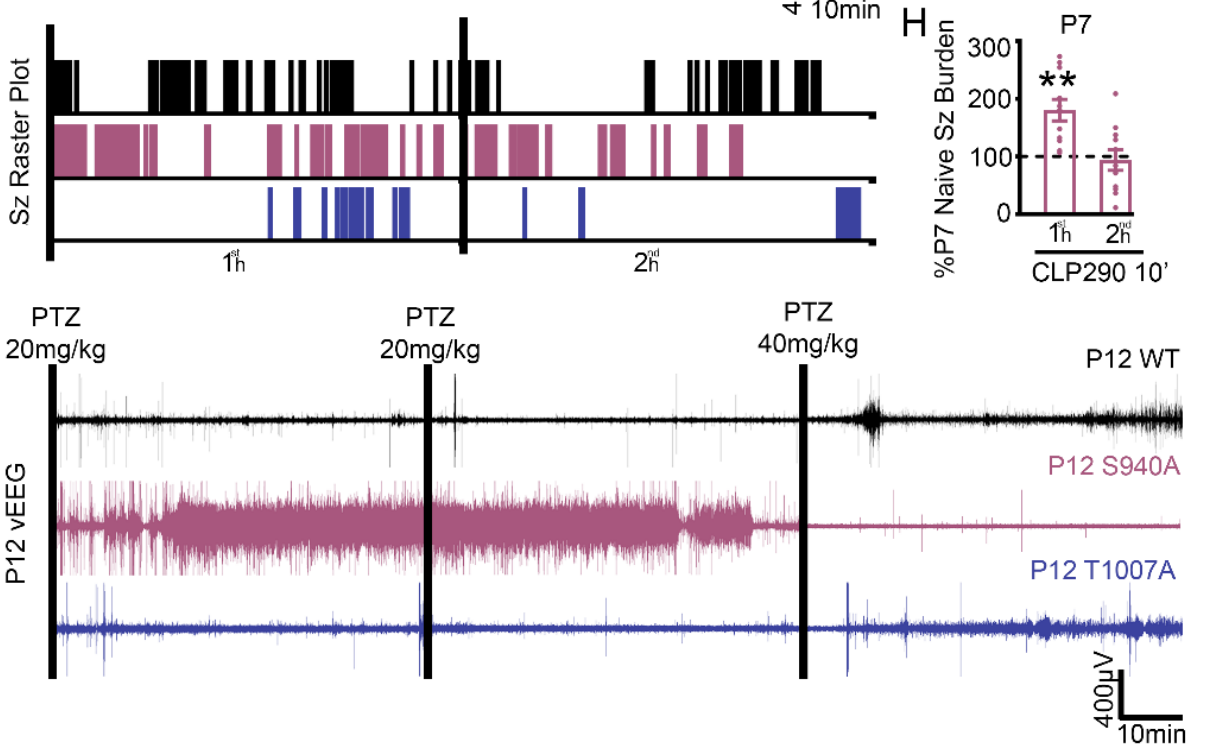

C
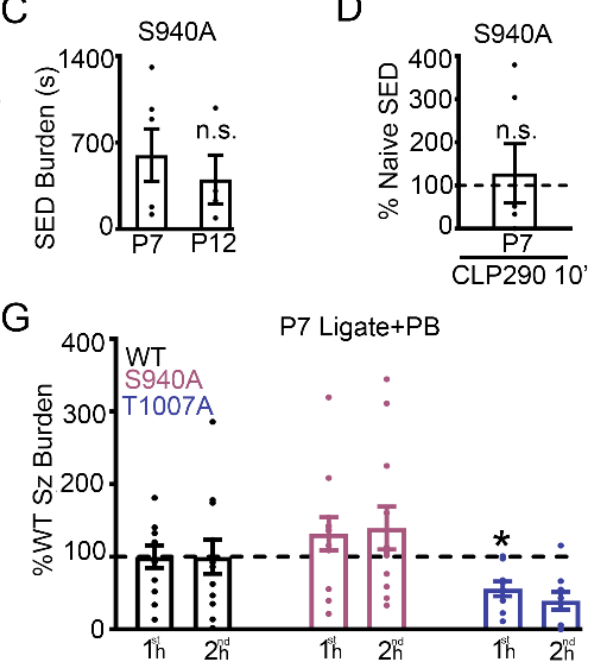

D

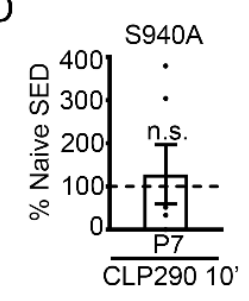

K

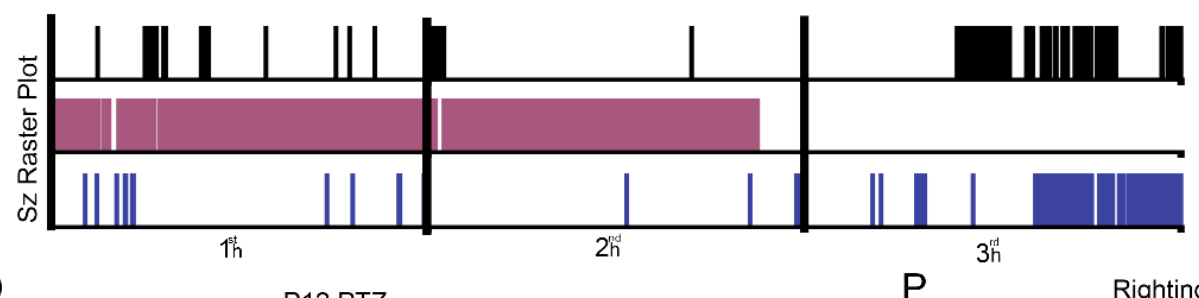

I 离 300 P10

N 200 in.s.

离 1000 南

요 P12

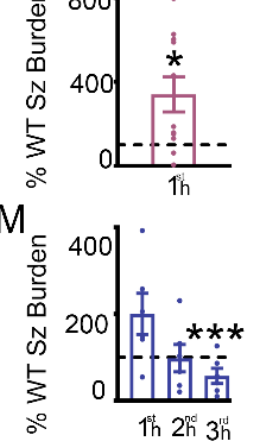

$\mathrm{N}$

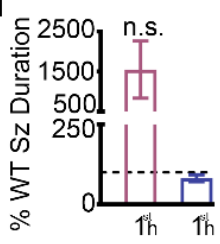

O

P12 PTZ
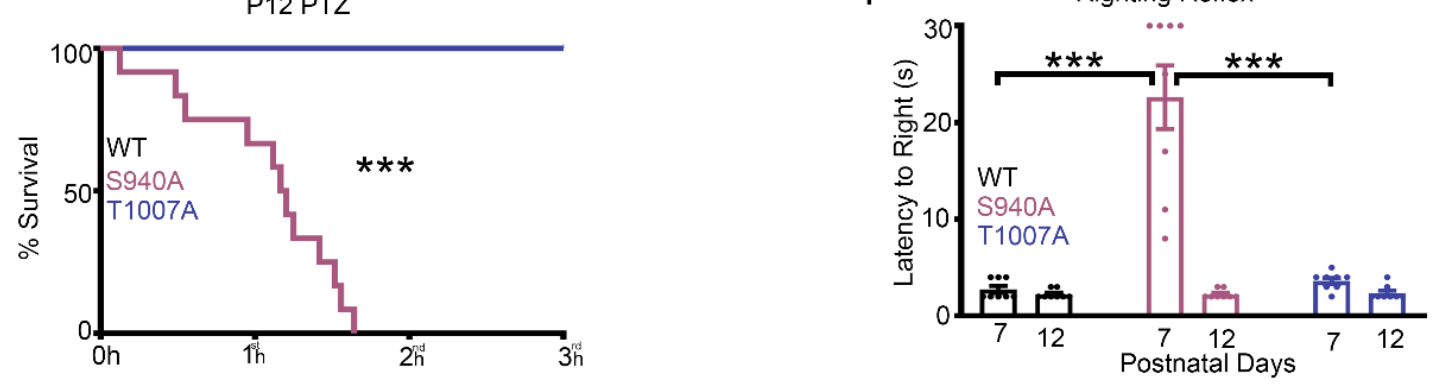
981 Fig.8. Inability to phosphorylate $\mathbf{S 9 4 0}$ or T1007 in knock-in pups regulated neonatal

982 seizure susceptibility. (A) Graphical representation of homozygous S940A+/+ knock-in

983 mutant mice (25) and homozygous T1007A knock-in mutant mice (24). (B) S940A+/+ mice

984 had spontaneous epileptiform discharges (SEDs) at P7 and (C) P12. (D) Spontaneous

985 epileptiform discharge duration of P7 S940A+/+ pups administered CLP290 10' treatment.

986 Naïve P7 S940A+/+ n=6; Naïve P12 S940A+/+ n=4; CLP290 10’ P7 S940A+/+ n=6. (E-G)

$987 \mathrm{P} \mathrm{T}_{1007 \mathrm{~A}^{+/+}}$pups are resistant to ischemic neonatal seizures. (E) EEG traces and (F)

988 seizure frequency raster plots for P7 WT, S940A ${ }^{+/+}$, and $\mathrm{T} 1007 \mathrm{~A}^{+/+}$pups that underwent

989 unilateral carotid ligation. (G) $1^{\text {st }}$ vs $2^{\text {nd }}$ hour seizure burdens after unilateral carotid

990 ligation plotted as percent WT. WT $n=12 ;$ S940A $A^{+/+} n=12 ;$ and $T 1007 A^{+/+} n=9$. (H) P7

991 S940A ${ }^{+/+}$ischemic seizures were CLP290 (10') resistant. Seizure burden of CLP290 10’

992 treated P7 S940A $^{+/+}$pups after unilateral carotid ligation plotted as P7 percent naïve

993 S940A $^{+/+}$. CLP290 10' treated P7 S940A ${ }^{+/+} \mathrm{n}=11$. (I) Seizure burden of P10 S940A S pups $^{+/+}$

$994(\mathrm{n}=8)$ after unilateral carotid ligation, plotted as P7 percent naïve S940A+/+. (J-O) Naïve

995 P12 S940A+/+ mice are susceptible to status epilepticus and mortality. (J) Representative

996 EEG trace and (K) seizure frequency raster plot of naïve P12 WT, S940A ${ }^{+/+}$, and

997 T1007A ${ }^{+/+}$pups. Black bars indicate intraperitoneal pentylenetetrazol (PTZ) injections. (L)

998 Total seizure burden of P12 S940A+/+ pups after PTZ administration plotted as percent

999 WT. (M) $1^{\text {st }}, 2^{\text {nd }}$, and $3^{\text {rd }}$ hour seizure burdens at $\mathrm{P} 12$ in $\mathrm{T}_{1007 \mathrm{~A}^{+/+}}$pups plotted as percent

1000 WT. (N) $1^{\text {st }}$ hour average seizure durations for S940A ${ }^{+/+}$and T1007A ${ }^{+/+} \mathrm{P} 12$ pups, plotted

1001 as percent WT. (O) Survival plot for P12 WT, T1007A+/+, and S940A $\mathrm{A}^{+/+}$pups during the

1002 PTZ challenge. P12 WT $n=11$; S940A ${ }^{+/+} n=11$; and $T 1007 A^{+/+} n=6$ pups. (P) Duration of

1003 time to righting reflex at P7 and P12 in naïve WT, T1007A+/+, and $S 940 A^{+/+}$pups. ( $\mathrm{n}=8$ 
1004 each group). ${ }^{*} \mathrm{P}<0.05 ;{ }^{* \star} \mathrm{P}<0.01 ;{ }^{* * *} \mathrm{P}<0.001$ by unpaired $\mathrm{t}$-test $\mathrm{vs}$. WT for all seizure data.

1005 Survival analysis ${ }^{* *} \mathrm{P}<0.001$ by Mantel-Cox test. Righting Reflex by one-way ANOVA.

1006

1007

1008

1009

1010

1011

1012

1013

1014

1015

1016

1017

1018

1019

1020

1021

1022

1023

1024

1025 


\section{Supplementary Materials for}

1027

Targeting ischemia-induced KCC2 hypofunction rescues refractory neonatal seizures and mitigates epileptogenesis in a mouse model

\section{Materials and Methods}

1036

\section{Unilateral carotid ligation}

A comprehensive protocol for unilateral carotid ligation and neonatal video-EEG

1040 unilateral ligation (without transection) of the right common carotid artery using 6-0 1041 surgisilk (Fine Science Tools, BC Canada) under isoflurane anesthesia. The outer skin 1042 was closed with 6-0 monofilament nylon (Covidien, MA), and lidocaine was applied as

1043 local anesthetic. Animals were implanted with 3 subdermal EEG scalp electrodes: 1 1044 recording and 1 reference overlying the bilateral parietal cortices, and 1 ground electrode 1045 overlying the rostrum. Wire electrodes (IVES EEG; Model \# SWE-L25 -MA, IVES EEG 1046 solutions, USA) were implanted subdermally and fixed in position with cyanoacrylate 1047 adhesive (KrazyGlue). Pups recovered from anesthesia over a few minutes. Animals 1048 were tethered to a preamplifier within a recording chamber for $2 \mathrm{~h}$ of continuous $\mathrm{VEEG}$ 
1049 recording and were maintained at $36^{\circ} \mathrm{C}$ with heated isothermal pads. At the end of the 1050 recording session, sub-dermal electrodes were removed, and the pups were returned to 1051 the dam.

1052

1053 CLP290 plasma and brain availability in neonatal mice

1054 Standards for HPLC were created using CLP257 (MilliporeSigma, USA) and 1055 CLP290 (Yves De Koninck Lab). P7 and P10 naïve pups of both sexes were administered 1056 CLP290 IP as three treatment groups: $10 \mathrm{mg} / \mathrm{kg}, 20 \mathrm{mg} / \mathrm{kg}$, or vehicle. After $4 \mathrm{~h}$ pups were 1057 anesthetized with chloral hydrate $(90 \mathrm{mg} / \mathrm{ml}$; IP), and transcardiac blood samples $(100 \mu \mathrm{L})$

1058 were collected. The same pups were transcardially perfused with ice cold PBS, and the 1059 whole fresh brains harvested. Brain samples were flash frozen in dry ice, homogenized 1060 using a sonicator, and stored at $-80^{\circ} \mathrm{C}$. Blood and brain samples from CLP290 treated 1061 and naïve pups were analyzed for CLP290 and CLP257 concentrations via HPLC using 1062 a C18 column and 10/90 organic/aqueous mobile phase.

1063

1064 VU 0463271

1065 To assess the role of KCC2 inhibition in neonatal seizure severity at P7 after 1066 ligation, the potent and selective KCC2 inhibitor VU 0463271 (VU) was administered at 1067 1h (0.5mg/kg; IP) in lieu of PB (Figure 1). VU was dissolved in 20/80\% dimethyl sulfoxide 1068 (DMSO)/PBS solution. To assess to role of KCC2 antagonism in neonatal seizure 1069 occurrence, naïve P7 or P10 pups underwent vEEG with $0.25 \mathrm{mg} / \mathrm{kg} \mathrm{VU}$ administered at 1070 the start of recording in lieu of ligation and $0.5 \mathrm{mg} / \mathrm{kg} \mathrm{VU}$ administered at $1 \mathrm{~h}$ in lieu of PB. 


\section{P12 PTZ Challenge}

To investigate the long-term effects of CLP290 treatment, P12 pups underwent a

1074 three hour vEEG recording during a pentylenetetrazole (PTZ; dissolved in 100\% PBS)

1075 challenge. At P7 pups were either naive, PB-only, or P7 CLP290 10'. These P7 pups then

1076 underwent a PTZ challenge at P12. All pups were administered PTZ at the beginning of

1077 the vEEG recording (20mg/kg; IP), at $1 \mathrm{~h}(20 \mathrm{mg} / \mathrm{kg} ; \mathrm{IP})$, and at $2 \mathrm{~h}(40 \mathrm{mg} / \mathrm{kg}$; IP).

1078

1079 Western blot analysis at 24h post-ligation

1080 All animals for immunochemical characterizations were anesthetized with chloral

1081 hydrate $(90 \mathrm{mg} / \mathrm{ml}$; IP) before being transcardially perfused with ice cold saline. The whole

1082 fresh brains were removed, the cerebellum was discarded, and the left and right

1083 hemispheres were separated. Brains were stored at $-80^{\circ} \mathrm{C}$ in preparation for further

1084 processing. Brain tissue homogenates were made and suspended in TPER cell lysis

1085 buffer containing $10 \%$ protease/phosphatase inhibitor cocktail. Total protein amounts

1086 were measured using the Bradford protein assay (Bio-Rad, Hercules, CA, USA) at 570nm

1087 and the samples diluted for $50 \mu \mathrm{g}$ of protein in each sample. $20 \mu \mathrm{L}$ of protein samples were

1088 run on $4-20 \%$ gradient tris-glycine gels (Invitrogen, Gand Island, NY, USA) for 120min at

$1089130 \mathrm{~V}$ and were transferred onto nitrocellulose membranes overnight at 20V. After the

1090 transfer, the nitrocellulose membranes underwent a $1 \mathrm{~h}$ blocking step in Rockland buffer

1091 before 6h incubation with primary antibodies (for all antibody RRIDS, see Key Resources

1092 Table): mouse $\alpha-K C C 2$ (1:1000, Millipore), rabbit $\alpha$-phospho-KCC2-S940 (1:1000 Aviva

1093 Systems Biology), rabbit $\alpha-p h o s p h o-K C C 2-T 1007$ (1:1000; Phospho solutions) mouse $\alpha-$

1094 TrkB (1:1000, BD Biosciences), rabbit a-phospho-TrkB-Y816 (1:500, Millipore), and 
1095 mouse a-actin (1:10000, LI-COR Biosciences). Membranes were then incubated with

1096 fluorescent secondary antibodies (1:5000, goat $\alpha$-rabbit and goat $\alpha$-mouse, Li-Cor

1097 Biosciences, USA). Chemiluminescent protein bands were analyzed using the Odyssey

1098 infrared imaging system 2.1 (LI-COR Biosciences). The optical density of each protein

1099 sample was normalized to their corresponding actin bands run on each lane for internal

1100 control. Mean normalized protein expression levels were then calculated for respective

1101 left and right hemispheres. The expression levels of the proteins of interest in ipsilateral

1102 hemispheres were normalized to the same in contralateral hemispheres for each pup to

1103 examine hemispheric percent change of protein expression.

1104

1105 Surface protein-seperation

$11061 \mathrm{~mm}$ coronal brain slices were obtained from P7 CD-1 mice and recovered for

$110745 \mathrm{~min}$ at $34^{\circ} \mathrm{C}$ with oxygenation $\left(95 \% / 5 \% \mathrm{O}_{2} / \mathrm{CO}_{2}\right)$. After recovery, slices were incubated 1108 with CLP290 or CLP257 at $34^{\circ} \mathrm{C}$ with oxygenation for $40 \mathrm{~min}$. Slices were placed in TPER

1109 cell lysing buffer with HALT protease and phosphatase inhibitors and homogenized via 1110 sonication. After 30min incubation on ice, protein lysates were ultracentrifuged at

1111 210,000xg (TLA-120.2 rotor, Beckman Coulter Life Sciences), and supernatants were

1112 collected as the cytosolic components. Pellets were resuspended in TPER/HALT buffer

1113 and ultracentrifuged; supernatant was discarded as wash fraction. Pellets were

1114 resuspended in TPER/HALT buffer and collected as the membrane components.

1115 Membrane and cytosolic components underwent Bradford analysis and Western blotting

1116 for protein quantification. Plasma membrane proteins were normalized to TfR. Cytosolic 1117 proteins were normalized to $\beta$-actin. 


\section{Supplemental Figure 1}

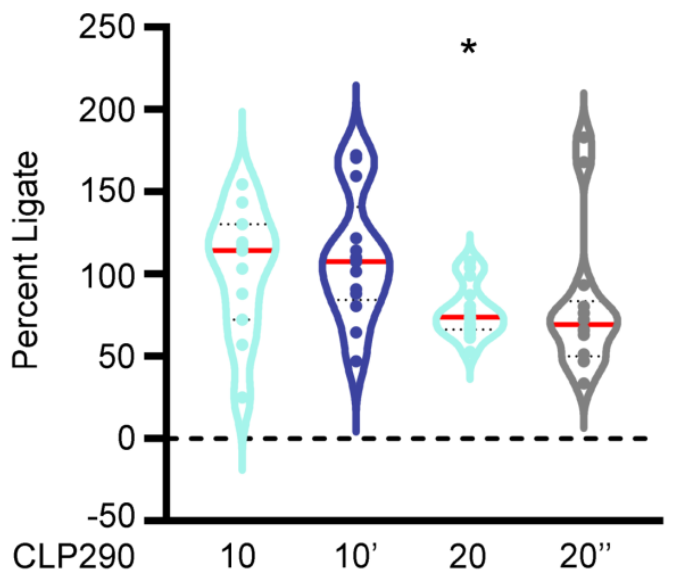


1135 Supplemental Fig. 1. CLP290 20 Post reduced first hour seizure burden. $1^{\text {st }}$ hour

1136 seizure burdens for 10,10 ',20, and 20" doses of CLP290. Violin plots show all data points

1137 as percent PB-only $1^{\text {st }}$ hour seizure burden. $\mathrm{P}=0.0477$, two-tailed $t$-test vs. PB-only $1^{\text {st }}$

1138 hour seizure burden.

1139

1140

1141

1142

1143

1144

1145

1146

1147

1148

1149

1150

1151

1152

1153

1154

1155

1156

1157 


\section{Supplemental Figure 2}

A

CLP290

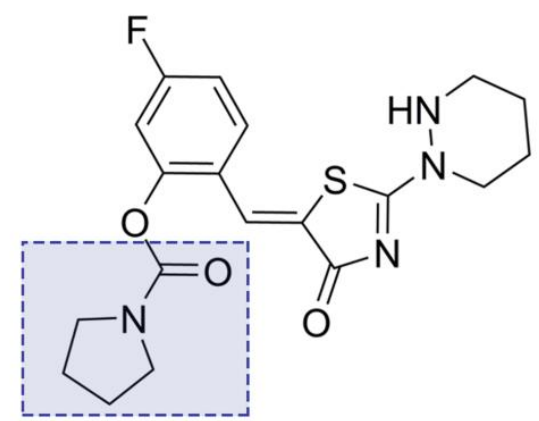

B

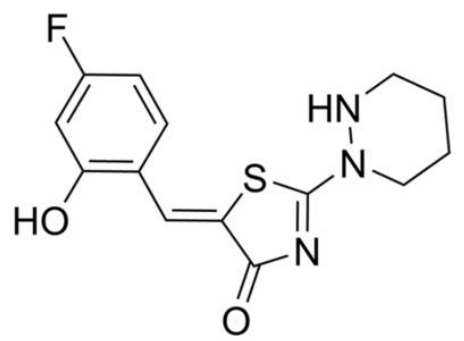

C

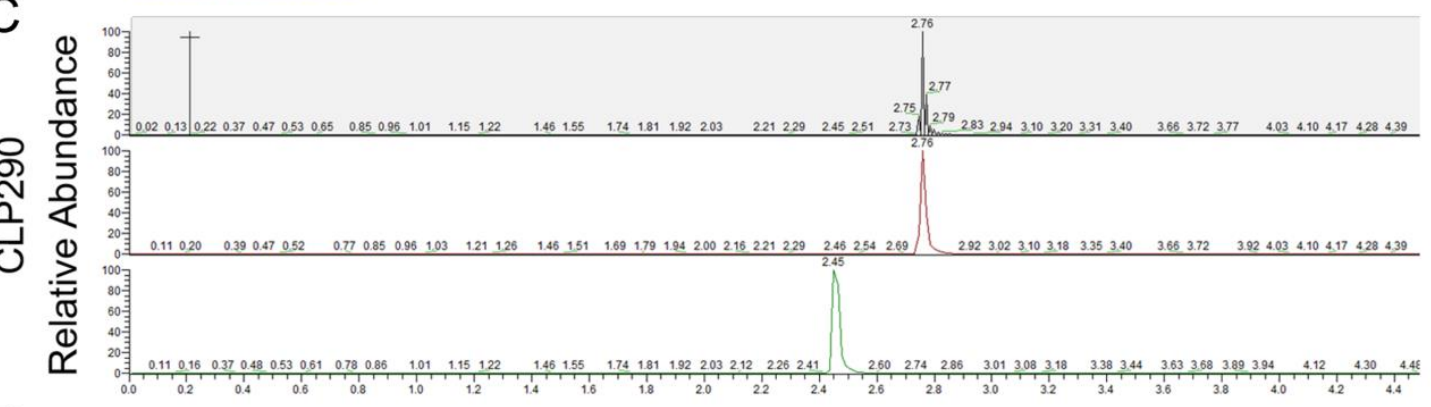

$\mathrm{D}$

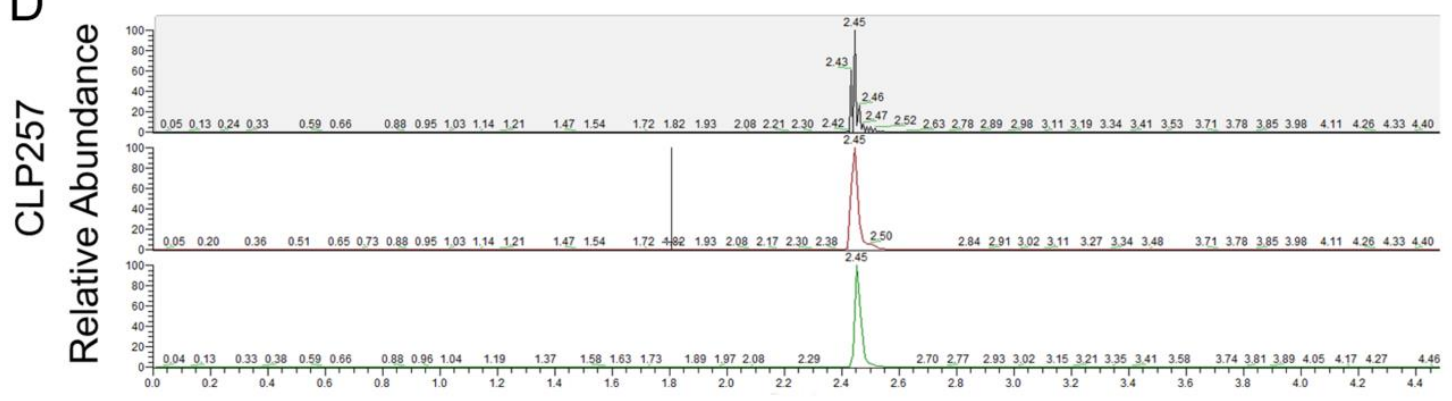

Time (min) 
1166 Supplemental Fig. 2. Characteristic peaks of CLP290 and CLP257 on HPLC. (A)

1167 CLP290 is the carbamate prodrug of (B) CLP257. (C) The characteristic peaks of CLP290

1168 (Pharmablock) and (D) CLP257 (Sigma) on HPLC.

1169

1170

1171

1172

1173

1174

1175

1176

1177

1178

1179

1180

1181

1182

1183

1184

1185

1186

1187

1188 
A

P7 S940 / KCC2

\#

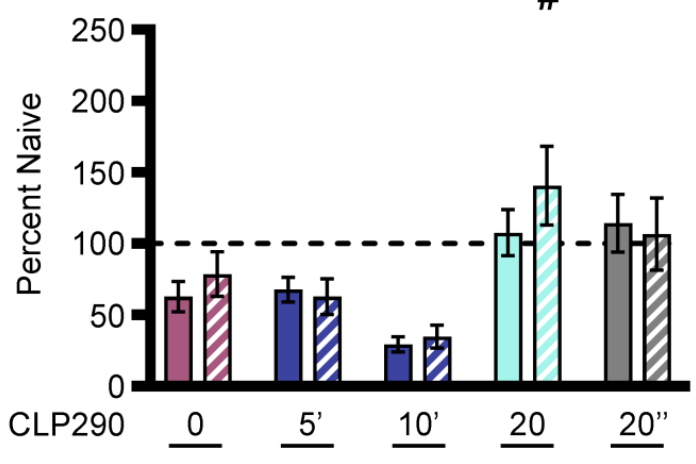

C

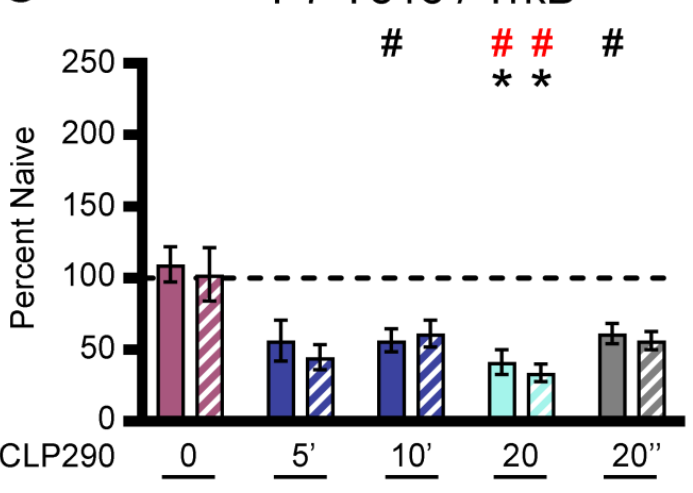

B $\quad \mathrm{P} 10 \mathrm{~S} 940 / \mathrm{KCC} 2$

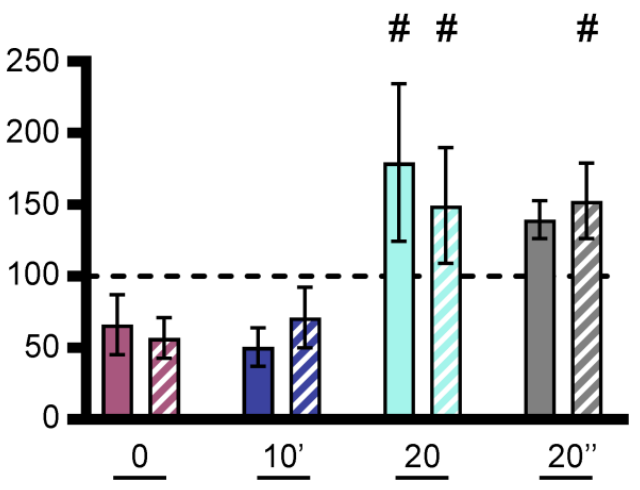

D $\quad$ P10 Y816 / TrkB

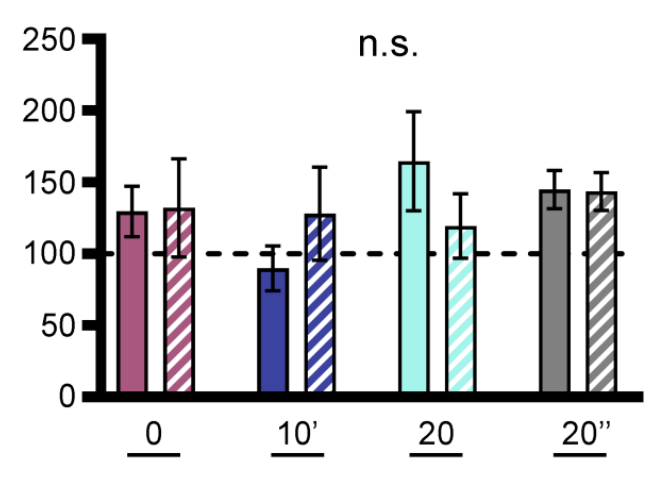


1201 Supplemental Fig. 3. CLP290 reduced Y816 activation. (A) S940/KCC2 ratios 24h

1202 after P7 ischemic neonatal seizures plotted as left and right hemispheres. (B)

1203 S940/KCC2 ratios $24 \mathrm{~h}$ after P10 ischemic neonatal seizures plotted as left and right

1204 hemispheres. (C) Y816/TrkB ratios 24h after P7 ischemic neonatal seizures plotted as

1205 left and right hemispheres. (D) Y816/TrkB ratios 24h after P10 ischemic neonatal seizures

1206 plotted as left and right hemispheres. ${ }^{*} \mathrm{P}<0.05$ and ${ }^{*} \mathrm{P}<0.001$ by 1 -way ANOVA vs. Naive.

$1207 \# P<0.05$ and $\# P<0.001$ vs. PB-only $P 7$ pups: Naïve $n=27$; PB-only $n=18 ; 5$ Post $n=3 ; 10$

1208 Post $n=9 ; 20$ Post $n=13 ; 5$ Primed $n=4 ; 10$ Primed $n=11 ; 20$ Pre $n=9$. P10 pups: Naïve

$1209 n=18$, PB-only $n=11,10$ Post $n=6,20$ Post $n=6,10$ Primed $n=5,20$ Pre $n=7$.

1210

1211

1212

1213

1214

1215

1216

1217

1218

1219

1220

1221

1222

1223 


\section{Supplemental Figure 4}

\section{T1007 \% R/L}

1225

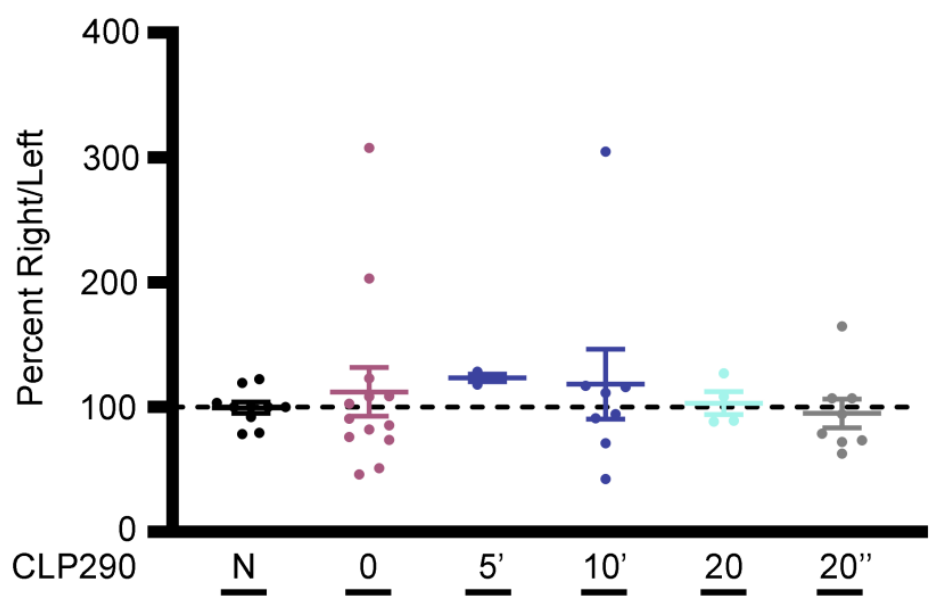


1239 Supplemental Fig. 4. T1007 is not activated by ischemia. T1007 expression as

1240 percent ipsilateral/contralateral plotted as percent naïve. 
bioRxiv preprint doi: https://doi.org/10.1101/2020.09.15.298596; this version posted September $15,2020$. The copyright holder for this preprint (which was not certified by peer review) is the author/funder. All rights reserved. No reuse allowed without permission.

\begin{tabular}{|c|c|c|c|c|}
\hline $\begin{array}{c}\text { Reagent type } \\
\text { (species) or resource }\end{array}$ & Designation & Source Reference & Identifiers & $\begin{array}{c}\text { Additional } \\
\text { Information }\end{array}$ \\
\hline $\begin{array}{c}\text { Genetic reagent (M. } \\
\text { musculus) }\end{array}$ & CD-1 & Charles River & 022 & $\mathrm{~N} / \mathrm{A}$ \\
\hline $\begin{array}{l}\text { Genetic reagent (M. } \\
\text { musculus) }\end{array}$ & S940A & Silayeva et al. 2015 & $\mathrm{~N} / \mathrm{A}$ & $\begin{array}{l}\text { Dr. Stephen Moss, } \\
\text { Tufts University } \\
\text { School of Medicine }\end{array}$ \\
\hline $\begin{array}{l}\text { Genetic reagent (M. } \\
\text { musculus) }\end{array}$ & T1007A & Moore et al. 2018 & $\mathrm{~N} / \mathrm{A}$ & $\begin{array}{l}\text { Dr. Stephen Moss, } \\
\text { Tufts University } \\
\text { School of Medicine }\end{array}$ \\
\hline $\begin{array}{c}\text { Chemical compound, } \\
\text { drug }\end{array}$ & Phenobarbital (PB) & MilliporeSigma & P5178 & $\mathrm{N} / \mathrm{A}$ \\
\hline $\begin{array}{l}\text { Chemical compound, } \\
\text { drug }\end{array}$ & CLP290 & Gagnon et al. 2013 & $\mathrm{~N} / \mathrm{A}$ & $\begin{array}{c}\text { Dr. Yves De } \\
\text { Koninck, Université } \\
\text { Laval }\end{array}$ \\
\hline $\begin{array}{c}\text { Chemical compound, } \\
\text { drug }\end{array}$ & CLP290 & PharmaBlock & PBSQ8214 & $\mathrm{N} / \mathrm{A}$ \\
\hline $\begin{array}{c}\text { Chemical compound, } \\
\text { drug }\end{array}$ & CLP257 & MilliporeSigma & SML1368 & $\mathrm{N} / \mathrm{A}$ \\
\hline $\begin{array}{l}\text { Chemical compound, } \\
\text { drug }\end{array}$ & $\begin{array}{l}\text { Pentylenetetrazol } \\
\text { (PTZ) }\end{array}$ & MilliporeSigma & P6500 & $\mathrm{N} / \mathrm{A}$ \\
\hline $\begin{array}{c}\text { Chemical compound, } \\
\text { drug }\end{array}$ & VU0463271 (VU) & Tocris & 4719 & $\mathrm{~N} / \mathrm{A}$ \\
\hline $\begin{array}{c}\text { Chemical compound, } \\
\text { drug }\end{array}$ & DMSO & Sigma & D8418 & $\mathrm{N} / \mathrm{A}$ \\
\hline $\begin{array}{l}\text { Chemical compound, } \\
\text { drug }\end{array}$ & HPCD & Sigma & $\mathrm{H} 107$ & $\mathrm{~N} / \mathrm{A}$ \\
\hline Software, algorithm & Graphpad Prism & Graphphad Software & $\begin{array}{c}\text { RRID:SCR_00279 } \\
8\end{array}$ & 8 \\
\hline Software, algorithm & Sirenia & Pinnacle Technology & $\begin{array}{c}\text { pinnaclet.com/sire } \\
\text { nia }\end{array}$ & $\begin{array}{c}\text { 3-Channel } \\
\text { EEG/EMG } \\
\text { Tethered Mouse } \\
\text { System }\end{array}$ \\
\hline Antibody & mouse $\alpha \mathrm{KCC} 2$ & $\begin{array}{c}\text { Aviva Systems Biology } \\
\text { OASE00240 }\end{array}$ & AB_2721238 & $1: 1000 ;$ WB \\
\hline Antibody & rabbit $\alpha$ pKCC2-S940 & $\begin{array}{c}\text { Aviva Systems Biology } \\
\text { OAPC00188 }\end{array}$ & AB_2721198 & 1:1000; WB \\
\hline Antibody & rabbit $\alpha$ pKCC2-1007 & $\begin{array}{l}\text { PhosphoSolutions } \\
\text { p1551-1007 }\end{array}$ & $A B \_2716769$ & 1:1000; WB \\
\hline Antibody & mouse $\alpha$ TrkB & $\begin{array}{l}\text { BD Biosciences } \\
610102\end{array}$ & AB_397508 & 1:1000; WB \\
\hline Antibody & rabbit $\alpha$ pTrkB-Y816 & Millipore ABN1381 & AB_2721199 & 1:500; WB \\
\hline Antibody & mouse $\alpha$ actin & $\begin{array}{l}\text { LI-COR Biosciences } \\
926-42213\end{array}$ & AB_2637092 & 1:10000; WB \\
\hline Antibody & $\begin{array}{l}\text { mouse } \alpha \text { Transferrin } \\
\text { Receptor }\end{array}$ & $\begin{array}{l}\text { ThermoFisher } \\
\text { Scientific }\end{array}$ & AB_2533029 & 1:500; WB \\
\hline Antibody & $\begin{array}{l}\text { goat } \alpha \text { mouse lgG, } \\
\text { IRDye } \AA 800 \mathrm{CW} \\
\text { Conjugated }\end{array}$ & $\begin{array}{l}\text { LI-COR Biosciences } \\
926-32210\end{array}$ & AB_621842 & 1:5000; WB \\
\hline Antibody & $\begin{array}{l}\text { goat } \alpha \text { rabbit IgG } \\
\text { Antibody, IRDye } \AA \\
\text { 680LT Conjugated }\end{array}$ & $\begin{array}{l}\text { LI-COR Biosciences } \\
926-68021\end{array}$ & AB_10706309 & 1:5000; WB \\
\hline
\end{tabular}

1253

1254 